\title{
, Maximum Average Service Rate and Optimal Queue 2 Scheduling of Delay-Constrained Hybrid Cognitive Radio in Nakagami Fading Channels
}

\author{
Jie Hu, Student Member, IEEE, Lie-Liang Yang, Senior Member, IEEE, and Lajos Hanzo, Fellow, IEEE
}

\begin{abstract}
5 Abstract-As a promising technique to improve achievable 6 bandwidth efficiency, cognitive radio (CR) has attracted substan7 tial research attention from both the academic and industrial com8 munities. To improve the performance attained by the secondary 9 user (SU), a novel hybrid CR system is proposed, which combines 10 the conventional interweave and underlay paradigms to enhance 11 the chance of the SU to access the spectrum. Queuing theory 12 is invoked in this paper to analyze the impact of the primary 13 user's maximum tolerable delay on the performance of the SU. 14 Multiple queues are assumed for the SU, which is engaged in 15 video communication. Apart from the Poisson traffic generation, 16 we also model the classic Nakagami- $\boldsymbol{m}$ fading channel as a Poisson 17 service process by utilizing the outage probability in the presence 18 of cochannel interference. We optimize both the hybrid inter19 weave/underlay procedure to maximize the average service rate $20 \mu_{S, \max }$ of the SU, as well as the queue's scheduling scheme, for the 21 sake of minimizing the overall average delay (OAD). As a result, 22 the OAD of the SU is reduced by up to $27 \%$ and $20 \%$, compared 23 with the proportion and round-robin schemes, respectively.
\end{abstract}

24 Index Terms-Hybrid cognitive radio (CR) system, maximum 25 average service rate (MASR) of secondary user (SU), multilayer 26 video streams (VSs), Nakagami- $m$ fading channel, optimal queue 27 scheduling scheme.

\section{INTRODUCTION}

29 WO kinds of customers are supported in cognitive radio 30 (CR), namely, the primary user (PU) and the secondary 31 user (SU). Furthermore, three main paradigms are consid32 ered for CR systems, namely, overlay, underlay, and inter33 weave paradigms [1]. Here, we focus our attention on the 34 interweave and underlay paradigms. According to the inter35 weave paradigm, the PUs are authorized to access the channel, 36 whereas the SUs are only able to access it when the PUs release 37 it. In the scenario that the PUs request resources, an SU's 38 session must be temporarily paused until the PUs complete 39 their transmission. In the interweave paradigm, SUs sense the 40 activities of PUs and clinch every possible opportunity to carry

Manuscript received June 21, 2012; revised November 15, 2012 and December 20, 2012; accepted December 27, 2012. This work was supported in part by the RC-UK's India-UK Advanced Technology Centre, by the European Union's Concerto Project, by the China-UK Science Bridge, and by the China Scholarship Council. The review of this paper was coordinated by Prof. C. Lin.

The authors are with the School of Electronics and Computer Science, University of Southampton, Southampton SO17 1BJ, U.K. (e-mail: jh10g11@ecs. soton.ac.uk; 1ly@ecs.soton.ac.uk; 1h@ecs.soton.ac.uk).

Color versions of one or more of the figures in this paper are available online at http://ieeexplore.ieee.org.

Digital Object Identifier 10.1109/TVT.2012.2237422

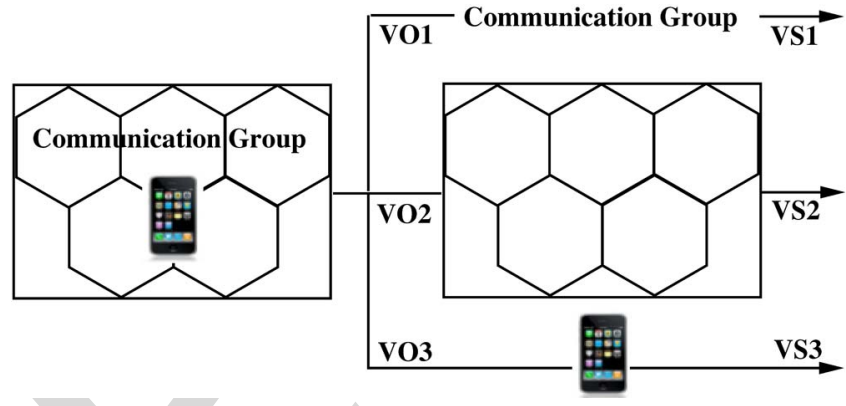

Fig. 1. Example of object-based MPEG-4 video transmission.

on with their own transmissions. By contrast, according to the 41 underlay paradigm, PUs and SUs are allowed to transmit their 42 data at the same time. An interference threshold is set up at 43 the PUs to guarantee that the PU's link is not unduly interfered 44 by SUs. Their transmission would only be terminated if the 45 interference imposed by SUs exceeds the threshold.

46

Although the transmission of PUs may be fully guaranteed 47 with the aid of the interweave paradigm, only limited op- 48 portunities may be offered to SUs to convey their own data. 49 Albeit SUs have improved chances of transmitting their own 50 information to the destination using the underlay paradigm, 51 the quality of service (QoS) of the PUs might not be as high 52 as for the interweave paradigm. Can we strike an attractive 53 compromise between these two paradigms so that the SUs have 54 more opportunities to access the channels while the PUs may 55 benefit from improved QoS? In this paper, a hybrid CR system 56 is invoked to achieve this goal. In our novel system, SUs first 57 detect the activity of PUs. If the channels are idle, the SUs 58 may access them to transmit their own data. By contrast, if 59 the channels are occupied by PUs, the SUs have a certain 60 probability of accessing the channels while ensuring that the 61 interference thresholds are not violated.

62

The Nakagami- $m$ channel model has the advantage of char- 63 acterizing a diverse range of multipath channels by a single 64 fading parameter $m$ [2]. Recently, supporting real-time video 65 services subject to specific QoS constraints has become one 66 of the essential requirements in wireless communications net- 67 works, where the video source is usually encoded into a number 68 of streams in the application layer, representing the base and 69 enhancement layers. An example of object-based MPEG-4 70 video transmission [3] is shown in Fig. 1, where different video 71 objects are encoded into different video streams (VSs). The 72 scheduling of the multilayer video queues at the SU's end 73 
74 deserves further attention for the sake of achieving the best 75 possible performance.

76 In [4], an adaptive resource-allocation scheme for multi77 layer VSs transmitted in wireless unicast/multicast scenarios is 78 proposed, which inspires our work. The maximum throughput 79 of an SU has been investigated in [5] under the interweave 80 paradigm by finding the optimal transmit power of the SU 81 in the scenario of a single queue conceived for the SU. In 82 [6], this research is extended to the multiple PU and single 83 SU CR system to explore the optimal transmit power and the 84 relaying probability at the SU. The stability region has been 85 already analyzed in the CR network supporting multiple PUs, 86 multiple relays, and multiple SUs [7]. In [5]-[7], the interweave 87 paradigm is employed, where the SUs have fewer opportunities 88 to access the resources. All these studies assumed, however, that 89 a single queue was set up for each of the users, which cannot 90 faithfully represent the characteristics of multimedia communi91 cation. Furthermore, all results have been generated assuming 92 queuing stability, where the specific QoS constraints such as 93 the delay tolerance of lip-synchronized video communications 94 were completely ignored.

95 Against this background, our novel contributions are as 96 follows.

97 1) We amalgamate the interweave and underlay paradigms 98 into a novel hybrid CR scheme characterized by the 99 parameter $0 \leq \varepsilon \leq 1$, which is zero when the pure inter100 weave paradigm is used and one for the pure underlay 101 paradigm.

102 2) The Nakagami- $m$ fading channel is used while modeling 103 the service as a Poisson process by deriving the closed117 including both the medium access control (MAC) and phys118 ical (PHY) layers, are described in Section II, whereas in 119 Section III, the related queuing analysis is carried out for both 120 the PU and the SU. In Section IV, the problem of finding the 121 optimal solution is formulated and solved, followed by our 122 numerical results in Section V. Finally, our conclusions are 123 offered in Section VI.

\section{SYSTEM MODEL}

125 Fig. 2 shows a CR system having a pair of PU source and 126 PU destination (PD), as well as a pair of SU source and SU 127 destination (SD). A novel hybrid CR policy is employed. Given 128 a spectrum band, the SU first senses the activity of the PU. If 129 this sensing process is reliable, the SU may access the spectrum

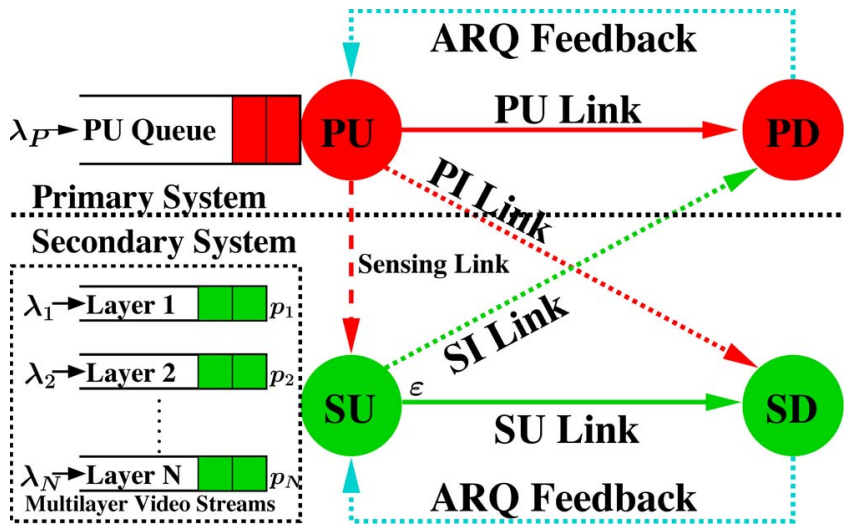

Fig. 2. System model with one PU and one SU sharing the same spectrum.

with a unity probability when the PU is idle. Furthermore, 130 the SU may access the spectrum with a probability of $0 \leq 131$ $\varepsilon \leq 1$, even if the PU is busy, provided that the PU's QoS is 132 still guaranteed. If, however, this sensing process failed, the 133 situation is reversed, and hence, the SU accesses the spectrum 134 with a probability of $\varepsilon$ when the PU is idle and with a unity 135 probability when the PU is busy. Hence, erroneous spectrum 136 sensing may lead to catastrophic performance degradation for 137 both the PU and the SU. We will also consider the practical 138 scenario of an erroneous spectrum-sensing decision and its 139 effect on the performance of the SU. Let us now consider the 140 details of the MAC and PHY layers.

\section{A. MAC-Layer Model}

Observe in Fig. 2 that a buffer is provided for the PU to store 143 the packets, which cannot be immediately transmitted. Again, 144 to support video communications for the SU, multiple buffers 145 are provided to accommodate the different-priority queues gen- 146 erated by multilayer video encoding.

The basic unit of time in our system is a time slot (TS). The 148 packets' arrival at the PU's buffer obeys the classic Poisson 149 process with a mean of $\lambda_{P}$ packets/TS. The QoS constraint 150 of the PU is the total delay imposed by both the transmission 151 and buffering delay, which should not exceed $T_{P}$, namely, the 152 maximum tolerable delay of the PU. The packets' arrival at 153 the buffer input of the SU also follows the Poisson process 154 with a mean of $\left\{\lambda_{n}, n=1,2, \ldots, N\right\}$ packets/TS. Regardless 155 of whether the PU is idle or busy, if the SU has already 156 successfully accessed the spectrum, we should determine the 157 probability of each queue at the SU completing its transmission 158 of the stored packets. These probabilities may be denoted 159 by $\left\{p_{n}, n=1,2, \ldots, N\right\}$. No specific QoS constraints are 160 imposed at the SU. However, our prime goal is to find the 161 optimal scheduling scheme and the associated hybrid parameter 162 $\varepsilon$, which minimizes the OAD of all the queues at the SU. 163

We assume that every packet transmitted by the PU or the 164 SU may be only successfully received by the corresponding 165 destination in a TS when the signal-to-interference-plus-noise 166 ratio (SINR) is above a specific threshold. In our system, 167 automatic repeat request (ARQ) with an unlimited number of 168 retransmissions is adopted to ensure that no packets are lost. 169 
170 Furthermore, we assume that the acknowledgements are always 171 successfully received by the transmitters.

172 Theorem 1: Given the successful reception probability $\mu$ of 173 a single packet during a TS in the ARQ-aided system, the 174 continuous transmit time obeys the exponential distribution 175 with the mean of $1 / \mu$.

176 Proof: The proof is provided in [8, App. A].

177 As a benefit of the ARQ mechanism, a packet's departure 178 from the buffer is synonymous to being served. The wireless 179 channel may be considered as a "server" in the queuing analy180 sis. As a result, the packets' departure process is also referred 181 to as the service process.

\section{B. PHY-Layer Model}

183 Observe from Fig. 2 that there are five links in this hybrid CR 184 system, namely, the PU link, the SU link, the sensing link, the 185 PI link imposing interference from the PU on the SD, and the 186 SI link inflicting interference from the SU upon the PD. In line 187 with [11], a multipacket reception (MPR) model is introduced 188 for the queuing analysis of the collision channel. We define the 189 corresponding conditional probabilities for the MPR model of 190 these five links.

$191 q_{\mathrm{PU} \mid\{\mathrm{PU}\}}^{(\mathrm{PD})}$ (or $q_{\mathrm{SU} \mid\{\mathrm{SU}\}}^{(\mathrm{SD})}$ ): Only the PU's (or SU's) packet is 192 successfully received at the PD (or SD) when only the PU 193 (or SU) transmits.

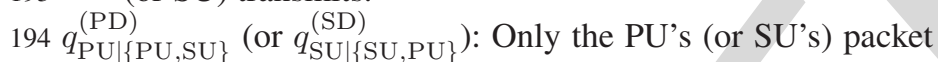
195 is successfully received at the PD (or SD) when both the $196 \quad$ PU and the SU transmit.

$197 q_{\mathrm{PU} \mid\{\mathrm{PU}\}}^{(\mathrm{SU})}$ : Only the PU's packet is successfully received at the 198 SU when the PU transmits.

199 Next, we will briefly highlight the derivation of these condi200 tional probabilities. The radio propagation between any pair of 201 nodes is assumed to be affected by the independent stationary 202 Nakagami- $m$ flat-fading channels $h_{i}(t)$ having $E\left[\left|h_{i}(t)\right|^{2}\right]=1$ 203 ( $t$ is the TS index). Hence, $Z=\left|h_{i}(t)\right|^{2}$ is a Gamma-distributed 204 random variable (r.v.) having a mean of 1, which may be 205 denoted by $Z \sim \operatorname{Gamma}(m, 1 / m)$ with the shape and scale 206 parameters of $m$ and $1 / m$, respectively. Given the probability 207 density function (pdf) $f_{Z}(z)$ of [9, eq. (3.39)], the tail dis208 tribution function (TDF) of $Z$ is formulated as $P(Z>z)=$ $209 \Gamma(m, m z) / \Gamma(m)$, where $\Gamma(\cdot, \cdot)$ represents the upper incomplete 210 Gamma function, and $m$ denotes the Nakagami- $m$ fading pa211 rameter [15].

212 After being divided by the noise power, the normalized trans213 mit power of the PU is defined as $P_{P}$, whereas the normalized 214 transmit power of the SU is defined as $P_{S}$. The propagation 215 path loss (PL) is denoted by $\Omega_{i}$, and the SINR threshold that 216 has to be exceeded for successful packet reception is denoted by $217 \beta_{i}$, while again, the Nakagami- $m$ fading parameter is denoted 218 by $m_{i}$, where $i$ represents "P" for the PU link, "S" for the SU 219 link, "PI" for the PI link, "SI" for the SI link, and "PS" for the 220 sensing link spanning from the PU to the SU.

221 According to the TDF of the r.v. $Z$, we express the con222 ditional probabilities of $q_{\mathrm{PU} \mid\{\mathrm{PU}\}}^{(\mathrm{PD})}, q_{\mathrm{SU} \mid\{\mathrm{SU}\}}^{(\mathrm{SD})}$, and $q_{\mathrm{PU} \mid\{\mathrm{PU}\}}^{(\mathrm{SU})}$ in the MPR model in the absence of interference thresholds as 223 follows:

$$
\begin{aligned}
q_{\mathrm{PU} \mid\{\mathrm{PU}\}}^{(\mathrm{PD})} & =P\left[\frac{\left|h_{P}(t)\right|^{2} P_{P}}{\Omega_{P}}>\beta_{P}\right] \\
& =\Gamma\left(m_{P}, \frac{m_{P} \beta_{P} \Omega_{P}}{P_{P}}\right) / \Gamma\left(m_{P}\right) \\
q_{\mathrm{SU} \mid\{\mathrm{SU}\}}^{(\mathrm{SD})} & =P\left[\frac{\left|h_{S}(t)\right|^{2} P_{S}}{\Omega_{S}}>\beta_{S}\right] \\
& =\Gamma\left(m_{S}, \frac{m_{S} \beta_{S} \Omega_{S}}{P_{S}}\right) / \Gamma\left(m_{S}\right) \\
q_{\mathrm{PU} \mid\{\mathrm{PU}\}}^{(\mathrm{SU})} & =P\left[\frac{\left|h_{\mathrm{PS}}(t)\right|^{2} P_{P}}{\Omega_{\mathrm{PS}}}>\beta_{\mathrm{PS}}\right] \\
& =\frac{\Gamma\left(m_{\mathrm{PS}}, m_{\mathrm{PS}} \beta_{\mathrm{PS}} \Omega_{\mathrm{PS}} / P_{P}\right)}{\Gamma\left(m_{\mathrm{PS}}\right)} .
\end{aligned}
$$

We introduce another two Gamma-distributed r.v.'s, namely, 225 $X \sim \operatorname{Gamma}\left(m_{X}, 1 / m_{X}\right)$ with a pdf of $f_{X}(x)$ and $Y \sim 226$ $\operatorname{Gamma}\left(m_{Y}, 1 / m_{Y}\right)$ with a pdf of $f_{Y}(y)$. Then, the problem 227 of solving $q_{\mathrm{PU} \mid\{\mathrm{PU}, \mathrm{SU}\}}^{(\mathrm{PD})}$ and $q_{\mathrm{SU} \mid\{\mathrm{SU}, \mathrm{PU}\}}^{(\mathrm{SD})}$ becomes equivalent to 228 solving the probability problem of $P[X>A+B Y]$. Unfortu- 229 nately, a closed-form solution cannot be derived for the general 230 situation. Nonetheless, we may obtain the following results for 231 two special cases:

$$
\begin{aligned}
& P[X>A+B Y] \\
& =\left\{\begin{array}{rr}
\Phi(A, B), & \text { if } m_{X} \text { is a positive integer } \\
& m_{Y} \text { can be any real number } \\
\Phi^{\prime}(A, B), & \text { if } m_{Y} \text { is a positive integer } \\
& m_{X} \text { can be any real number. }
\end{array}\right. \\
& \left\{\begin{aligned}
\Phi(A, B)= & \left(\frac{m_{Y}}{m_{X} B+m_{Y}}\right)^{m_{Y}} e^{-m_{X} A} \\
& \cdot \sum_{n=0}^{m_{X}-1} \sum_{k=0}^{n} \frac{m_{X}^{n} B^{k} A^{n-k}}{k !(n-k) !\left(m_{X} B+m_{Y}\right)^{k}} \frac{\Gamma\left(m_{Y}+k\right)}{\Gamma\left(m_{Y}\right)} \\
\Phi^{\prime}(A, B)= & \frac{\Gamma\left(m_{X}, m_{X} A\right)}{\Gamma\left(m_{X}\right)}-\sum_{n=0}^{m_{Y}-1} \sum_{k=0}^{n}\left(\begin{array}{l}
n \\
k
\end{array}\right)\left(\frac{m_{Y}}{B}\right)^{n} \\
& \cdot \frac{e^{m_{Y} \frac{A}{B}(-A)^{n-k} m_{X}^{m_{X}}}}{n ! \Gamma\left(m_{X}\right)} \cdot \frac{\Gamma\left[k+m_{X}, A\left(m_{X}+m_{Y} / B\right)\right]}{\left(m_{X}+m_{Y} / B\right)^{k+m_{X}}} .
\end{aligned}\right.
\end{aligned}
$$

For a detailed derivation of $\Phi(A, B)$ and $\Phi^{\prime}(A, B)$, see 233 [8, App. B]. Given this result, we may write down the other 234 two conditional probabilities when subjected to the cochannel 235 interference thresholds at the PD and the SD as follows:

236

$$
\begin{aligned}
& q_{\mathrm{PU} \mid\{\mathrm{PU}, \mathrm{SU}\}}^{(\mathrm{PD})} \\
& \quad=P\left[\frac{\left|h_{P}(t)\right|^{2} P_{P} / \Omega_{P}}{1+\left|h_{\mathrm{SI}}(t)\right|^{2} P_{S} / \Omega_{\mathrm{SI}}}>\beta_{P}\right] \\
& \quad=P\left[\left|h_{P}(t)\right|^{2}>\frac{\beta_{P} \Omega_{P}}{P_{P}}+\frac{\beta_{P} P_{S} \Omega_{P}}{P_{P} \Omega_{\mathrm{SI}}}\left|h_{\mathrm{SI}}(t)\right|^{2}\right] \\
& \quad= \begin{cases}\Phi\left(\frac{\beta_{P} \Omega_{P}}{P_{P}}, \frac{\beta_{P} P_{S} \Omega_{P}}{P_{P} \Omega_{\mathrm{SI}}}\right), & \text { if } m_{P} \text { is a positive integer } \\
\Phi^{\prime}\left(\frac{\beta_{P} \Omega_{P}}{P_{P}}, \frac{\beta_{P} P_{S} \Omega_{P}}{P_{P} \Omega_{S I}}\right), & \text { if } m_{S I} \text { is a positive integer }\end{cases}
\end{aligned}
$$




$$
\begin{aligned}
& q_{\mathrm{SU} \mid\{\mathrm{SU}, \mathrm{PU}\}}^{(\mathrm{SD})} \\
& \quad=P\left[\frac{\left|h_{S}(t)\right|^{2} P_{S} / \Omega_{S}}{1+\left|h_{\mathrm{PI}}(t)\right|^{2} P_{P} / \Omega_{\mathrm{PI}}}>\beta_{S}\right] \\
& =P\left[\left|h_{S}(t)\right|^{2}>\frac{\beta_{S} \Omega_{S}}{P_{S}}+\frac{\beta_{S} P_{P} \Omega_{S}}{P_{S} \Omega_{\mathrm{PI}}}\left|h_{\mathrm{PI}}(t)\right|^{2}\right] \\
& \quad= \begin{cases}\Phi\left(\frac{\beta_{S} \Omega_{S}}{P_{S}}, \frac{\beta_{S} P_{P} \Omega_{S}}{P_{S} \Omega_{\mathrm{PI}}}\right), & \text { if } m_{S} \text { is a positive integer } \\
\Phi^{\prime}\left(\frac{\beta_{S} \Omega_{S}}{P_{S}}, \frac{\beta_{S} P_{P} \Omega_{S}}{P_{S} \Omega_{\mathrm{PI}}}\right), & \text { if } m_{\mathrm{PI}} \text { is a positive integer. }\end{cases}
\end{aligned}
$$

\section{QUEUING ANALYSIS}

238 The following tasks will be accomplished here: 1) The classic $239 \mathrm{M} / \mathrm{M} / 1$ queuing is revisited. 2) The average service rate of the $240 \mathrm{PU}$ and the SU is attained for the original benchmark system. $2413)$ A new system is defined for characterizing the interdepen242 dence of the PU's and SU's queues, and the corresponding 243 average service rate of the PU and the SU is rederived.

\section{A. $M / M / 1$ Queuing System}

245 In the $M / M / 1$ queuing system, only a single server is in246 voked by the system. The packets' interarrival time follows 247 the exponential distribution having a parameter $\lambda$, whereas the 248 packets' interdeparture time obeys the same distribution having 249 a parameter $\mu$. An infinite buffer is assumed for guaranteeing 250 that every single packet may be successfully transmitted, i.e., 251 without any packet loss. According to [10], we can express 252 the probability of the queue being empty and the total average 253 delay, including the transmission and buffering time, as follows:

$$
P[Q=0]=1-\lambda / \mu, \quad T=1 /(\mu-\lambda), \quad \text { where } \quad \lambda<\mu
$$

254 and we denote the random queue length and the total average 255 delay by $Q$ and $T$, respectively.

\section{B. PU Link}

257 According to the MAC-layer protocol of our hybrid CR 258 system, the PU has the highest priority to access the channel. If 259 the PU's queue is not empty, the PU would occupy the channel 260 for its transmission, and the packet would be removed from the 261 head of the queue when it is successfully received by the PD. 262 However, the PU would suffer from the interference imposed 263 by the activity of the SU. The service process of a PU's single 264 packet follows the Bernoulli distribution. In analogy to the 265 successful packet departure probability in the Bernoulli trial, 266 the average service rate of the Poisson service process may be 267 formulated for the PU link as

$$
\begin{aligned}
\mu_{P}= & P\left\{Q_{S}=0\right\} \cdot q_{\mathrm{PU} \mid\{\mathrm{PU}\}}^{(\mathrm{PD})}+P\left\{Q_{S} \neq 0\right\} \\
& \cdot\left(q_{\mathrm{PU} \mid\{\mathrm{PU}\}}^{(\mathrm{SU})} \cdot\left(q_{\mathrm{PU} \mid\{\mathrm{PU}\}}^{(\mathrm{PD})} \cdot(1-\varepsilon)+q_{\mathrm{PU} \mid\{\mathrm{PU}, \mathrm{SU}\}}^{(\mathrm{PD})} \cdot \varepsilon\right)\right. \\
& +\left(1-q_{\mathrm{PU} \mid\{\mathrm{PU}\}}^{(\mathrm{SU})}\right) \cdot q_{\mathrm{PU} \mid\{\mathrm{PU}, \mathrm{SU}\}}^{(\mathrm{PD})}
\end{aligned}
$$

where $Q_{S}$ represents the random queue length of the SU. Given 268 the average service rate $\mu_{P}$, the average arrival rate $\lambda_{P}$, and the 269 QoS constraint stipulating that the total delay should not exceed 270 the maximum delay tolerance $T_{P}$, we infer from (4) that the 271 condition $0 \leq \lambda_{P} \leq \mu_{P}-1 / T_{P}$ must be obeyed.

\section{SU Link}

According to the MAC-layer protocol of the SU, before 274 accessing the channel, the SU carries out spectrum sensing first. 275 When the channel is released by the PU, after reliable sensing, 276 the SU would transmit its own packets with a probability 277 of unity (otherwise, with a probability of $\varepsilon$ ), which implies 278 inefficient resource usage. When the channel is still occupied 279 by the PU, following reliable sensing, the SU would transmit 280 its own packets with a probability of $\varepsilon$ (otherwise, with a 281 probability of unity), which imposes strong interference on the 282 PD. It is vital to guarantee that the transmission of the SU does 283 not force the PU to violate its delay tolerance. We derive the 284 probability of a packet being served, which is also identical to 285 the average service rate $\mu_{S}$ of the SU's Poisson service process 286 expressed as

$$
\begin{aligned}
\mu_{S}= & q_{\mathrm{SU} \mid\{\mathrm{SU}\}}^{(\mathrm{SD})} \cdot P\left[Q_{P}=0\right] \\
& \cdot\left[q_{\mathrm{PU} \mid\{\mathrm{PU}\}}^{(\mathrm{SU})}+\left(1-q_{\mathrm{PU} \mid\{\mathrm{PU}\}}^{(\mathrm{SU})}\right) \cdot \varepsilon\right] \\
& +q_{\mathrm{SU} \mid\{\mathrm{SU}, \mathrm{PU}\}}^{(\mathrm{SD})} \cdot P\left[Q_{P} \neq 0\right] \\
& \cdot\left[q_{\mathrm{PU} \mid\{\mathrm{PU}\}}^{(\mathrm{SU})} \cdot \varepsilon+\left(1-q_{\mathrm{PU} \mid\{\mathrm{PU}\}}^{(\mathrm{SU})}\right)\right]
\end{aligned}
$$

where $Q_{P}$ represents the random queue length of the PU.

\section{Stochastic Dominance Principle}

It may be shown from (5) and (6) that the average service 290 rates of the SU and the PU depend on each other's queue sizes. 291 Since these queues interact with each other, the average rate of 292 the individual service processes cannot be directly determined. 293 For the sake of circumventing this problem, the stochastic 294 dominance principle of [12] is invoked to assist our analysis. 295

The concept of a so-called "dominant system" was defined 296 in [12] by allowing a set of terminals having no packets in their 297 transmit-buffer to continue transmitting hypothetical dummy 298 packets. In this manner, the queues in the dominant system 299 stochastically dominate the queues in the original system. This 300 dominant system is defined here.

301

1) If we have $Q_{S}=0$ and $Q_{P}=0$, the SU transmits dummy 302 packets with a unity probability.

303

2) If $Q_{S}=0$ and $Q_{P} \neq 0$, the SU transmits dummy packets 304 with a probability $\varepsilon$.

305

Note that the hypothetical dummy packets are introduced 306 only for the sake of facilitating our performance analysis and 307 for finding closed-form solutions to the optimum scheduling 308 factor $\varepsilon$, but in reality, no dummy packets are assumed to 309 be transmitted in the practical systems considered. As shown 310 in [13], given the same initial conditions, the queuing per- 311 formance of the dominant system transmitting hypothetical 312 
313 dummy packets is capable of providing a tight approximation 314 of the queuing performance of the original system. Since in 315 practical systems no dummy packets are actually transmitted, 316 no extra interference will be imposed on the system, and no 317 extra power is required for their transmissions.

318 In the context of the extended dominant system, $\left\{Q_{S} \neq 0\right\}$ 319 has a unity probability. Hence, (5) can be rewritten as

$$
\begin{array}{r}
\mu_{P}=q_{\mathrm{PU} \mid\{\mathrm{PU}\}}^{(\mathrm{SU})} \cdot\left(q_{\mathrm{PU} \mid\{\mathrm{PU}\}}^{(\mathrm{PD})} \cdot(1-\varepsilon)+q_{\mathrm{PU} \mid\{\mathrm{PU}, \mathrm{SU}\}}^{(\mathrm{PD})} \cdot \varepsilon\right) \\
+\left(1-q_{\mathrm{PU} \mid\{\mathrm{PU}\}}^{(\mathrm{SU})}\right) \cdot q_{\mathrm{PU} \mid\{\mathrm{PU}, \mathrm{SU}\}}^{(\mathrm{PD})}
\end{array}
$$

320 and the condition $0 \leq \lambda_{P} \leq \mu_{P}-1 / T_{P}$ is also rewritten as

$$
\begin{aligned}
& 0 \leq \lambda_{P} \leq q_{\mathrm{PU} \mid\{\mathrm{PU}\}}^{(\mathrm{SU})} q_{\mathrm{PU} \mid\{\mathrm{PU}\}}^{(\mathrm{PD})} \\
&+\left(1-q_{\mathrm{PU} \mid\{\mathrm{PU}\}}^{(\mathrm{SU})}\right) q_{\mathrm{PU} \mid\{\mathrm{PU}, \mathrm{SU}\}}^{(\mathrm{PD})}-1 / T_{P} \\
& \quad-q_{\mathrm{PU} \mid\{\mathrm{PU}\}}^{(\mathrm{SU})}\left(q_{\mathrm{PU} \mid\{\mathrm{PU}\}}^{(\mathrm{PD})}-q_{\mathrm{PU} \mid\{\mathrm{PU}, \mathrm{SUh}\}}^{(\mathrm{PD})}\right) \varepsilon .
\end{aligned}
$$

321 According to (4), we may represent the probabilities of the PU's 322 queue being not empty and empty by $\lambda_{P}$ and $\mu_{P}$, respectively, 323 as shown in (7). Hence, we may rewrite (6) as

$$
\begin{aligned}
\mu_{S}=q_{\mathrm{SU} \mid\{\mathrm{SU}\}}^{\mathrm{SD}} & q_{\mathrm{PU} \mid\{\mathrm{PU}\}}^{(\mathrm{SU})}+q_{\mathrm{SU} \mid\{\mathrm{SU}\}}^{\mathrm{SD}}\left(1-q_{\mathrm{PU} \mid\{\mathrm{PU}\}}^{(\mathrm{SU})}\right) \varepsilon \\
-\frac{\lambda_{P}}{\mu_{P}} & {\left[q_{\mathrm{SU} \mid\{\mathrm{SU}\}}^{\mathrm{SD}} q_{\mathrm{PU} \mid\{\mathrm{PU}\}}^{(\mathrm{SU})}+q_{\mathrm{SU} \mid\{\mathrm{SU}\}}^{\mathrm{SD}}\right.} \\
& \times\left(1-q_{\mathrm{PU} \mid\{\mathrm{PU}\}}^{(\mathrm{SU})}\right) \varepsilon-q_{\mathrm{SU} \mid\{\mathrm{SU}, \mathrm{PU}\}}^{\mathrm{SD}} q_{\mathrm{PU} \mid\{\mathrm{PU}\}}^{(\mathrm{SU})} \varepsilon \\
& \left.-q_{\mathrm{SU} \mid\{\mathrm{SU}, \mathrm{PU}\}}^{\mathrm{SD}}\left(1-q_{\mathrm{PU} \mid\{\mathrm{PU}\}}^{(\mathrm{SU})}\right)\right]
\end{aligned}
$$

324 To simplify the derivations in the next section, some sim325 ple notations are introduced as follows: $C=q_{\mathrm{PU} \mid\{\mathrm{PU}\}}^{(\mathrm{PD})}, D=$ $326 q_{\mathrm{PU} \mid\{\mathrm{PU}, \mathrm{SU}\}}^{(\mathrm{PD})}, E=q_{\mathrm{SU} \mid\{\mathrm{SU}\}}^{(\mathrm{SD})}, F=q_{\mathrm{SU} \mid\{\mathrm{SU}, \mathrm{PU}\}}^{(\mathrm{SD})}$, and $S=q_{\mathrm{PU} \mid\{\mathrm{PU}\}}^{(\mathrm{SU})}$

\section{7}

\section{Problem Formulation and Solutions}

328 Here, the following two problems are solved: 1) finding the 329 optimal $\varepsilon^{*}$ for maximizing the average service rate $\mu_{S, \max }$ of 330 the SU and 2) finding the optimal queue scheduling scheme $331\left\{p_{n}^{*}, n=1,2, \ldots, N\right\}$ to minimize the OAD associated with $332 \mu_{S, \max }$.

\section{A. MASR of the $S U$}

334 The optimal parameter $\varepsilon^{*}$ will be found for the sake of 335 maximizing the average service rate of the SU without violating 336 the delay tolerance of the PU. The solution can be found by 337 constructing the problem $\mathbf{P 1}$ as

$$
\begin{gathered}
\arg \max _{\varepsilon} \mu_{S}(\varepsilon) \\
0 \leq \varepsilon \leq \xi=\frac{S C+(1-S) D-\lambda_{P}-1 / T_{P}}{S(C-D)} \leq 1
\end{gathered}
$$

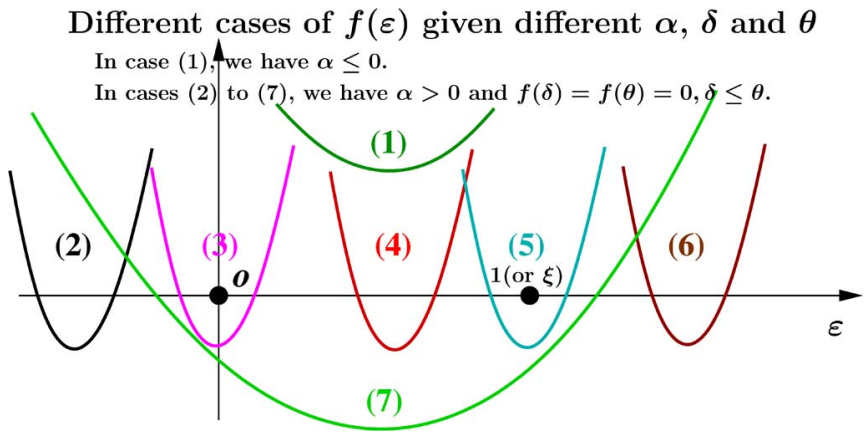

Fig. 3. Different cases of $f(\varepsilon)$ given different $\alpha, \delta$, and $\theta$.

where the function $\mu_{S}(\varepsilon)$ is defined by (9), whereas the inequal- 338 ity condition in (10) is imposed for the sake of satisfying the 339 specific delay tolerance of the PU, which is derived from (8). 340

To solve P1, we differentiate $\mu_{S}(\varepsilon)$ with respect to $\varepsilon, 341$ yielding

$$
\frac{d \mu_{S}}{d \varepsilon}=\frac{f(\varepsilon)}{[S C+(1-S) D-S(C-D) \varepsilon]^{2}}
$$

where we define $f(\varepsilon)=E(1-S)[S C+(1-S) D-S(C-343$ $D) \varepsilon]^{2}-\lambda_{P} \alpha$ and $\alpha=S(C-D)(E-F)+D E(1-S)-344$ $F S D$. Since the denominator of (11) is always positive, we 345 can simply consider $f(\varepsilon)$ instead, which is a quadratic function 346 having the shape shown in Fig. 3.

347

Let us consider the constraint shown in (8) more closely. 348 Given $0 \leq \varepsilon \leq 1$, the right side of (8) reaches its minimum 349 value of $\left(D-1 / T_{P}\right)$ when we have $\varepsilon=1$. Therefore, if we 350 have $\lambda_{P} \leq D-1 / T_{P}, \varepsilon$ may assume any arbitrary value rang- 351 ing from 0 to 1 . By contrast, if $\lambda_{P}>D-1 / T_{P}$, then $\varepsilon$ must 352 be selected from the range determined by (10) in the context of 353 the specific $\lambda_{P}$ value.

Different scenarios have to be considered for finding the 355 optimal values of $\varepsilon^{*}$, which lead to the maximum average 356 service rate (MASR) $\mu_{S}(\varepsilon)$ of the SU formulated in (10). $\quad 357$

Case $1\left(\lambda_{P} \leq D-1 / T_{P}\right)$ : In this case, as aforementioned, 358 $\varepsilon$ might assume any arbitrary value ranging from 0 to 1 . Under 359 this assumption, the problem may be further divided into the 360 following two subcases, which will assist us in finding $\mu_{S, \max } .361$

Subcase 1-1 $(\alpha \leq 0)$ : When $\alpha \leq 0$, the $f(\varepsilon)$ curve obeys the 362 relative position shown by case (1) at the top of Fig. 3, in which 363 case there are no real-valued solutions to $f(\varepsilon)=0$. Hence, 364 provided that $E(1-S)>0$ holds, $f(\varepsilon)$ is always higher than 365 0 , implying that $\mu_{S}(\varepsilon)$ is a monotonically increasing function. 366 Therefore, we have $\varepsilon^{*}=1$ and $\mu_{S, \max }=\mu_{S}(1)$.

Subcase 1-2 $(\alpha>0)$ : Second, when $\alpha>0$, there are two 368 real-valued solutions to $f(\varepsilon)=0$, which are denoted by $\delta$ and 369 $\theta$, respectively, i.e.,

$$
\begin{aligned}
\delta= & \frac{1}{S(C-D)} \\
& {[S C+(1-D)} \\
& -\sqrt{\left.\frac{\lambda_{P}[S(C-D)(E-F)+D E(1-S)-F S D]}{E(1-S)}\right]}
\end{aligned}
$$




$$
\begin{aligned}
\theta= & \frac{1}{S(C-D)} \\
& {[S C+(1-D)} \\
& +\sqrt{\left.\frac{\lambda_{P}[S(C-D)(E-F)+D E(1-S)-F S D]}{E(1-S)}\right]}
\end{aligned}
$$

371 where we have $\delta \leq \theta$. In this case, depending on the values of $\delta$ 372 and $\theta$, the relative position of the $f(\varepsilon)$ curve corresponds to one 373 of the scenarios (2)-(7) shown in Fig. 3, which are specifically 374 considered in the following.

a) If $\delta \leq \theta<0$, the $f(\varepsilon)$ curve obeys the positioning of case (2) in Fig. 3, where $f(\varepsilon)>0$ holds within the range of $0 \leq \varepsilon \leq 1$, implying that $\mu_{S}(\varepsilon)$ is a monotonically increasing function of $\varepsilon$. Hence, $\varepsilon^{*}=1$ maximizes the average service rate $\mu_{S}(\varepsilon)$, which is $\mu_{S, \max }=\mu_{S}(1)$.

b) If $\delta<0$ and $0 \leq \theta<1$, the $f(\varepsilon)$ curve obeys the positioning of case (3) in Fig. 3. Hence, $f(\varepsilon) \leq 0$ holds within the range of $0 \leq \varepsilon \leq \theta$, which implies that $\mu_{S}(\varepsilon)$ is a monotonically decreasing function of $\varepsilon$. However, within the range of $\theta<\varepsilon \leq 1, f(\varepsilon)>0$ holds, and hence, $\mu_{S}(\varepsilon)$ is a monotonically increasing function of $\varepsilon$. In this situation, the optimal value of $\varepsilon^{*}$ is either 0 or 1 . Hence, we have $\varepsilon^{*}=\arg \max _{\varepsilon \in\{0,1\}}\left\{\mu_{S}(\varepsilon)\right\}$, and the MASR becomes $\mu_{S, \max }=\max \left\{\mu_{S}(0), \mu_{S}(1)\right\}$.

c) If $0 \leq \delta<\theta<1$, the $f(\varepsilon)$ curve corresponds to case (4) in Fig. 3. Therefore, $f(\varepsilon) \geq 0$ holds within the range of $0 \leq \varepsilon \leq \delta$, implying that $\mu_{S}(\varepsilon)$ is a monotonically increasing function of $\varepsilon$. Within the range of $\delta<\varepsilon \leq$ $\theta$, however, $f(\varepsilon) \leq 0$ holds, which suggests that $\mu_{S}(\varepsilon)$ is a monotonically decreasing function of $\varepsilon$. Finally, in the range of $\theta<\varepsilon \leq 1, f(\varepsilon)>0$ is satisfied, which indicates that $\mu_{S}(\varepsilon)$ becomes a monotonically increasing function of $\varepsilon$ again. According to the preceding analysis, the pair of potential values that may maximize $\mu_{S}(\varepsilon)$ are $\varepsilon=\delta$ and $\varepsilon=1$. Hence, the optimal value of $\varepsilon^{*}$ is $\varepsilon^{*}=\arg \max _{\varepsilon \in\{\delta, 1\}}\left\{\mu_{S}(\varepsilon)\right\}$, and the MASR is, hence, $\mu_{S, \max }=\max \left\{\mu_{S}(\delta), \mu_{S}(1)\right\}$.

d) If $0 \leq \delta<1$ and $\theta \geq 1$, which results in an $f(\varepsilon)$ curve corresponding to case (5) in Fig. 3, $f(\varepsilon) \geq 0$ holds within the range of $0 \leq \varepsilon \leq \delta$, which implies that $\mu_{S}(\varepsilon)$ is a monotonically increasing function of $\varepsilon$. Within the range of $\delta<\varepsilon \leq 1$, however, $f(\varepsilon)<0$ holds, which implies that $\mu_{S}(\varepsilon)$ is a monotonically decreasing function of $\varepsilon$. Jointly considering these two situations, we conclude that $\varepsilon^{*}=\delta$ maximizes the average service rate $\mu(\varepsilon)$, which is $\mu_{S, \max }=\mu_{S}(\delta)$.

e) If $\delta \geq 1$, we have encountered case (6) of Fig. 3. Correspondingly, $f(\varepsilon) \geq 0$ holds within the range of $0 \leq \varepsilon \leq$ 1 , which implies that $\mu_{S}(\varepsilon)$ is a monotonically increasing function of $\varepsilon$. Hence, $\varepsilon^{*}=1$ maximizes the average service rate of $\mu_{S}(\varepsilon)$, which is $\mu_{S, \max }=\mu_{S}(1)$.

f) Finally, if $\delta<0<1 \leq \theta, f(\varepsilon)$ is reminiscent of case (7) of Fig. 3. Consequently, $f(\varepsilon) \leq 0$ holds within the range of $0 \leq \varepsilon \leq 1$, which implies that $\mu_{S}(\varepsilon)$ is a monotoni- cally decreasing function of $\varepsilon$. Hence, $\varepsilon^{*}=0$ maximizes 419 the average service rate $\mu_{S}(\varepsilon)$, which is $\mu_{S, \max }=\mu_{S}(0) .420$

Case $2\left(\lambda_{P}>D-1 / T_{P}\right)$ : If $\lambda_{P}>D-1 / T_{P}$, then $\varepsilon$ may 421 assume any arbitrary value ranging from 0 to $\xi$, where the value 422 of $\xi$ is restricted by the value of $\lambda_{P}$ according to (10). Following 423 a similar train of thought, by simply substituting "1" in Case 1424 by " $\xi$," we have the following conclusions, which correspond 425 to the cases (1)-(7) in Fig. 3, as we discussed earlier. 426

1) If $\alpha \leq 0$ (see case (1) in Fig. 3), or if $\alpha>0$ and $\theta<0427$ (see case (2) in Fig. 3), or if $\alpha>0$ and $\xi \leq \delta$ (see case (6) 428 in Fig. 3), then we have $\varepsilon^{*}=\xi$, and the MASR becomes 429 $\mu_{S, \max }=\mu_{S}(\xi)$.

2) If $\alpha>0, \delta<0$, and $0 \leq \theta<\xi$ (see case (3) in Fig. 3), 431 then we have $\varepsilon^{*}=\arg \max _{\varepsilon \in\{0, \xi\}}\left\{\mu_{S}(\varepsilon)\right\}$, and the 432 MASR becomes $\mu_{S, \max }=\max \left\{\mu_{S}(0), \mu_{S}(\xi)\right\}$.

433

3) If $\alpha>0$ and $0 \leq \delta<\theta<\xi$ (see case (4) in Fig. 3), then 434 we have $\varepsilon^{*}=\arg \max _{\varepsilon \in\{\delta, \xi\}}\left\{\mu_{S}(\varepsilon)\right\}$, and the MASR is 435 $\mu_{S, \max }=\max \left\{\mu_{S}(\delta), \mu_{S}(\xi)\right\}$.

436

4) If $\alpha>0$ and $0 \leq \delta<\xi \leq \theta$ (see case (5) in Fig. 3), then 437 $\varepsilon^{*}=\delta$, and the MASR is $\mu_{S, \max }=\mu_{S}(\delta)$.

438

5) If $\alpha>0, \delta<0$, and $0<\xi \leq \theta$ (see case (7) in Fig. 3), then 439 $\varepsilon^{*}=0$, and the MASR is formulated as $\mu_{S, \max }=\mu_{S}(0) .440$

\section{B. Optimal Queue Scheduling of the $S U$}

441

Let us now further detail the derivation of the optimal queue 442 scheduling scheme $\left\{p_{n}^{*}, n=1,2, \ldots, N\right\}^{1}$ conceived for min- 443 imizing the OAD of the SU's queues. Given (4), the average 444 delay of each queue may be formulated as

445

$$
\operatorname{Delay}_{n}=1 /\left(p_{n} \cdot \mu_{S}(\varepsilon)-\lambda_{n}\right), \quad n=1,2, \ldots, N
$$

where $p_{n}$ is the probability of the SU's $n$th queue transmitting 446 its stored packets, and $\lambda_{n}$ is the packets' average arrival rate. 447

Given the associated MASR $\mu_{S, \text { max }}$ of the SU, we construct 448 the second problem $\mathbf{P 2}$, i.e.

449

$$
\begin{aligned}
& \arg \min _{\left\{p_{1}, p_{2}, \ldots, p_{N}\right\}}\left\{\frac{1}{N} \sum_{n=1}^{N} \frac{1}{p_{n} \cdot \mu_{S, \max }-\lambda_{n}}\right\} \\
& \text { subject to } \sum_{n=1}^{N} p_{n}=1, \quad \lambda_{n}-p_{n} \cdot \mu_{S, \max }<0 .
\end{aligned}
$$

Then, we may readily show the following.

1) The objective function (15) is convex over $\left\{p_{n}, n=451\right.$ $1,2, \ldots, N\}$.

2) The first equality constraint in (16) is affine over 453 $\left\{p_{n}, n=1,2, \ldots, N\right\}$, whereas the second inequality 454 constraint in (16) is convex over $\left\{p_{n}, n=1,2, \ldots, N\right\} .455$

Hence, $\mathbf{P} \mathbf{2}$ is a convex problem [14], and the optimal solution 456 may be obtained with the aid of the Lagrangian optimization 457 method while additionally exploiting the Karush-Kuhn-Tucker 458 (KKT) conditions [14], which were proposed for solving 459 convex optimization problems similar to ours under an inequal- 460 ity constraint.

461

\footnotetext{
${ }^{1}$ The optimal shceduling scheme is fully specified when the probability $p_{n}$ of transmitting the stream $n$ is determined.
} 
TABLE I

PHY-LAYER PARAMETER SETTING

\begin{tabular}{|l|r|r|r|r|}
\hline & $\mathrm{PL}$ & Threshold & Fading & Norm Power \\
\hline PU Link & $\Omega_{P}=5 \mathrm{~dB}$ & $\beta_{P}=13.9$ or $8.5 \mathrm{~dB}$ & $m_{P}=1$ or 2 & $P_{P}=26 \mathrm{~dB}$ \\
\hline SU Link & $\Omega_{S}=5 \mathrm{~dB}$ & $\beta_{S}=13.9$ or $8.5 \mathrm{~dB}$ & $m_{S}=1$ or 2 & $P_{S}=26 \mathrm{~dB}$ \\
\hline PI/SI Link & $\Omega_{P I / S I}=15 \mathrm{~dB}$ & No threshold & $m_{S I / P I}=1$ & $P_{P / S}=26 \mathrm{~dB}$ \\
\hline Sensing Link & $\Omega_{P S}=2 \mathrm{~dB}$ & $\beta_{P S}=13.9$ or $8.5 \mathrm{~dB}$ & $m_{P S}=1$ or 2 & $P_{P}=26 \mathrm{~dB}$ \\
\hline
\end{tabular}

462

$$
\begin{aligned}
J=\frac{1}{N} \sum_{n=1}^{N} \frac{1}{p_{n} \mu_{S, \max }-\lambda_{n}} \\
\quad+\sum_{n=1}^{N} \varphi_{n}\left(\lambda_{n}-p_{n} \mu_{S, \max }\right)+\eta\left(\sum_{n=1}^{N} p_{n}-1\right)
\end{aligned}
$$

463 where $\eta \geq 0$ and $\varphi_{n} \geq 0, n=1,2, \ldots, N$ are the Lagrangian 464 multipliers associated with the two conditions of (16), respec465 tively. Then, the optimal queue scheduling scheme $\left\{p_{n}^{*}, n=\right.$ $4661,2, \ldots, N\}$ and the Lagrangian multipliers of the optimization 467 problem P2 satisfy the following KKT conditions [14]:

$$
\left\{\begin{array}{l}
\left.\frac{\partial J}{\partial p_{n}}\right|_{p_{n}=p_{n}^{*}}=\frac{-\mu_{S, \max }}{N\left(p_{n}^{*} \mu_{S, \max }-\lambda_{n}\right)^{2}}-\varphi_{n}^{*} \mu_{S, \max }+\eta^{*}=0 \\
\left.\frac{d J}{d \eta}\right|_{p_{n}=p_{n}^{*}}=\sum_{n=1}^{N} p_{n}^{*}-1=0 \\
\varphi_{n}^{*}\left(\lambda_{n}-p_{n}^{*} \mu_{S, \max }\right)=0, \quad \varphi_{n}^{*} \geq 0 \text { and } \eta^{*} \geq 0
\end{array}\right.
$$

468 where $n=1,2, \ldots, N$. Naturally, according to the second con469 straint of (16), the only solution satisfying the third line of (18) 470 is $\varphi_{n}^{*}=0$. Substituting $\varphi_{n}^{*}=0$ into the first line of (18), we 471 derive $p_{n}^{*}$ as

$$
p_{n}^{*}=\frac{1}{\sqrt{N \eta^{*} \mu_{S, \max }}}+\frac{\lambda_{n}}{\mu_{S, \max }} .
$$

472 Then, substituting (19) into the second line of (18), we arrive at

$$
\eta^{*}=N \cdot \mu_{S, \max } /\left(\mu_{S, \max }-\sum_{n=1}^{N} \lambda_{n}\right)^{2}
$$

473 Finally, substituting $\eta^{*}$ of (20) into (19), we arrive at the optimal 474 queue scheduling scheme characterized by

$$
p_{n}^{*}=\frac{\mu_{S, \max }-\sum_{n=1}^{N} \lambda_{n}+N \lambda_{n}}{N \cdot \mu_{S, \max }} .
$$

\section{5 \\ V. PERFormance AnAlysis}

476 In our numerical results, we link the PHY-layer parameters to 477 a realistic orthogonal frequency-division multiplexing scheme 478 communicating over Nakagami- $m$ fading channels without 479 channel coding. We define the SINR threshold of the receiver 480 for the sake of guaranteeing that the bit error ratio of the system 481 is not higher than $10^{-2}$. Hence, the threshold is determined 482 by the channel model having different fading parameters. For 483 example, according to [16, Fig. 5-5], if $m=1$, the threshold 484 at the receiver is $13.86 \mathrm{~dB}$, whereas the threshold is $8.50 \mathrm{~dB}$ 485 for $m=2$.

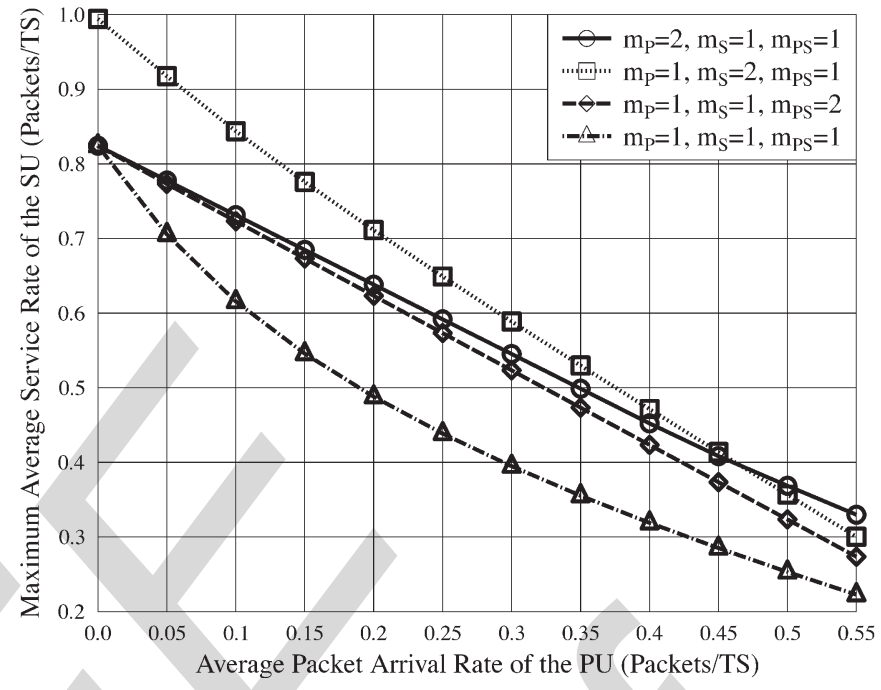

Fig. 4. Service rate of the SU versus the average packets arrival rate $\lambda_{P}$ of the PU parameterized by the fading parameters.

We assume that the SU has five different queuing buffers 486 in the MAC layer, where we set the average packet arrival 487 rate of each queue, for example, to $0.01,0.02,0.03,0.04$, and 488 0.05 packet/TS, respectively.

Two other queue scheduling schemes are used as benchmark- 490 ers, namely, the round-robin scheme, where every queue of the 491 SU has the same probability of transmitting packets stored in 492 the buffer, and the proportional scheme, where the transmission 493 probabilities are arranged to be proportional to the average 494 arrival rates at the buffers.

A. Impact of the PU'S Average Packet Arrival Rate

496

In the PHY layer, the PL, the SINR threshold of the receiver 497 associated with the Nakagami- $m$ fading parameters, and the 498 transmit power are set according to Table I. More particu- 499 larly, there are four cases for the Nakagami- $m$ parameters of 500 the PU, the SU, and the sensing links, namely, 1) $m_{P}=1,501$ $m_{S}=1$, and $m_{P S}=1$; 2) $m_{P}=2, m_{S}=1$, and $m_{P S}=1 ; 502$ 3) $m_{P}=1, m_{S}=2$, and $m_{P S}=1$; and 4) $m_{P}=1, m_{S}=1,503$ and $m_{P S}=2$.

In the MAC layer, the delay tolerance of the PU is $T_{P}=505$ $5 \mathrm{TS}$. We vary the average packet arrival rate $\lambda_{P}$ from 0.1 to 506 0.5 packet/TS for characterizing its impacts on the MASR and 507 the OAD in conjunction with three different queue scheduling 508 schemes for the SU.

It may be readily shown from Fig. 4 that $\mu_{S, \max }$ lin- 510 early decays upon increasing $\lambda_{P}$. This is plausible since a 511 higher $\lambda_{P}$ results in an increased traffic load for the PU link, 512 which, in turn, results in the PU's prolonged occupation of the 513 
514 spectral band. This reduces the SU's chance of transmitting its 515 own packets, which is reflected by the reduction of $\mu_{S, \max }$. 516 Upon increasing the Nakagami- $m$ parameters of the PU, SU, 517 and sensing links, respectively, the MASR is improved. If 518 we consider the higher $m_{P}$ of the PU link, it is shown in 519 Fig. 4 that the data stream of the PU may be transmitted more 520 promptly and the duration of the PU's spectrum occupation 521 would be shortened. Consequently, the SU's increased chances 522 of transmitting its own data results in an increased MASR. 523 Hence, the MASR is substantially improved. If we consider the 524 higher $m_{S}$ of the SU link characterized in Fig. 4, the MASR is 525 also directly improved. For higher $m_{P S}$ values, the MASR of 526 Fig. 4 would be also improved due to the fact that the spectrum527 sensing decisions become more reliable.

528 We may infer the following observations from Fig. 4 depend529 ing on the traffic load of the PU. We have the best performance 530 gain upon improving the SU link if the PU's traffic load is 531 lower than 0.45 packet/TS. By contrast, if the traffic load is 532 higher than 0.45 packet/TS, improving the PU link may provide 533 the best performance. Upon comparing the improvements of 534 the sensing and PU links shown in Fig. 4, when the PU's 535 traffic load is lower than 0.1 packet/TS, we infer that improving 536 the sensing link may provide the same performance gain as 537 improving the PU link. However, when the traffic load is higher 538 than 0.1 packet/TS, improving the PU link is more influential. 539 These observations may motivate us to design a more effective 540 strategy for the sake of improving the performance of the SU, 541 given different traffic loads of the PU. For example, given a 542 light PU traffic load, improving the SU link is our best option 543 to enhance overall performance. Furthermore, a reliable sensing 544 strategy conceived for the SU may achieve the same overall 545 performance as an improved PU link. By contrast, for a high 546 traffic load, researchers should focus on directly improving the 547 PU link.

\section{B. Impact of the PU'S Delay Tolerance on the $S U$}

549 Here, we consider the Nakagami- $m$ parameters of $m_{P}=$ $550 m_{S}=m_{P S}=2$, and all the associated SINR thresholds are $5518.5 \mathrm{~dB}$, where the transmit power of the PU and the SU are $552 P_{P}=P_{S}=18 \mathrm{~dB}$, whereas the others are the same as in 553 Table I. We vary the delay tolerance $T_{P}$ from 3 to 8 TS for 554 the sake of investigating its impacts on the OAD in conjunction 555 with three different queue scheduling schemes. Two scenarios, 556 namely, $\lambda_{P}=0.4$ and 0.5 packet/TS, are considered.

557 Observe furthermore in Fig. 5 that the SU benefits from 558 increasing $T_{P}$ and that a reduced $\lambda_{P}$ decreases the OAD. The 559 asymptotic values of the OAD for these three schemes may 560 be determined when $T_{P}$ tends to infinity. In Fig. 5, we focus 561 our attention on the comparison of three different scheduling 562 schemes. It can be seen that our proposed optimal scheme 563 (OPT) performs better than the conventional schemes. The 564 higher the traffic load of the PU, i.e., the higher $\lambda_{P}$ and 565 lower $T_{P}$, the more substantial the advantage of our optimal 566 scheme becomes. For example, for $\lambda_{P}=0.5$ packet/slot and $567 T_{P}=3 \mathrm{TS}$, our scheme has a $27 \%$ or $20 \%$ lower OAD than 568 the proportional (PRO) or the round-robin (R-R) schemes, 569 respectively, when considering the SU's queues.

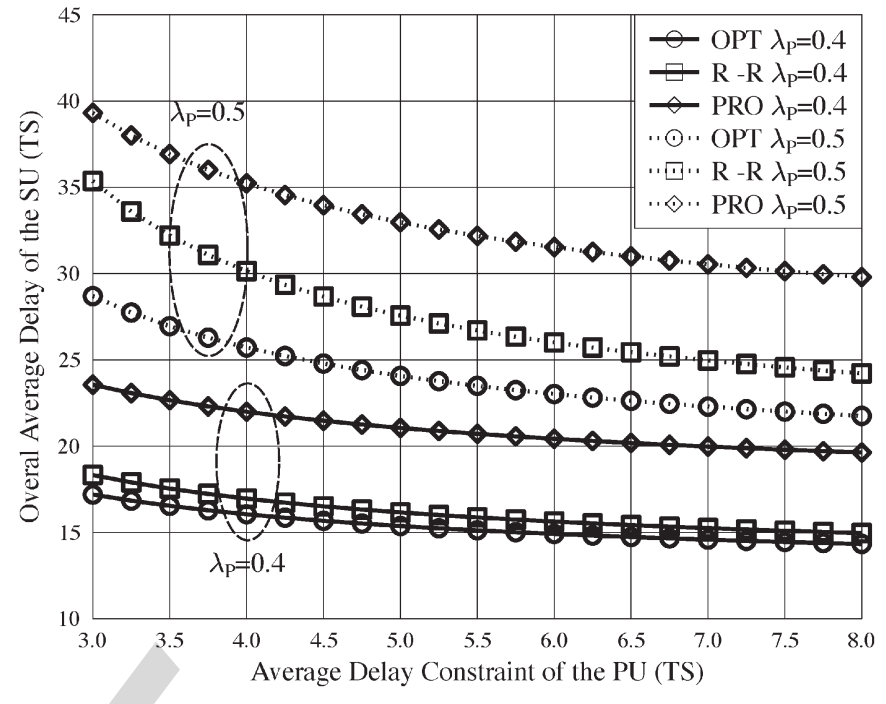

Fig. 5. Delay of the SU versus the delay tolerant $T_{P}$ of the PU parameterized by the average packets arrival rate $\lambda_{P}$.

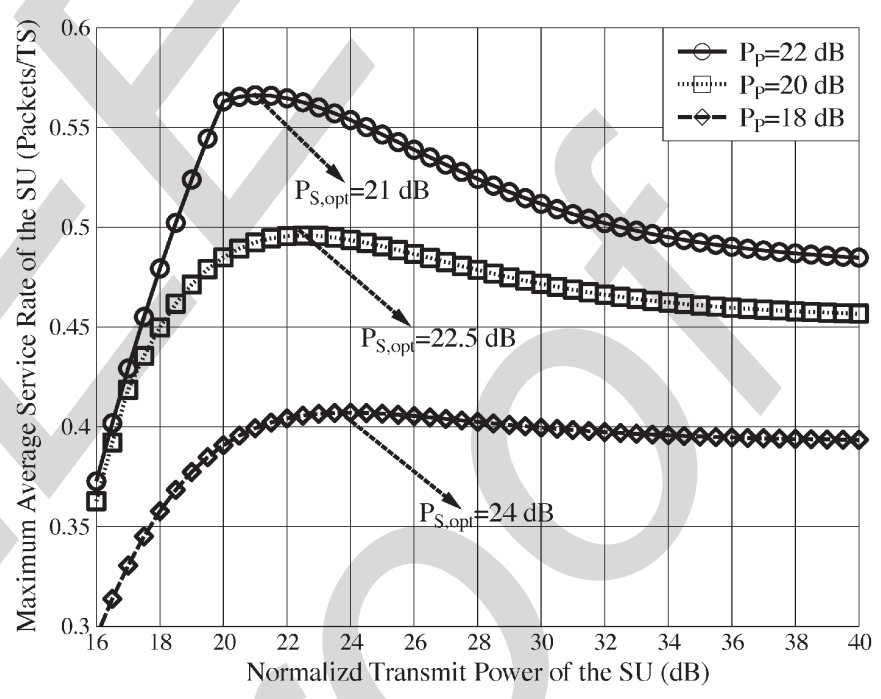

Fig. 6. Service rate versus the normalized transmit power of the SU parameterized by the normalized transmit power of the PU.

C. Impact of the SU's Normalized Transmit Power

In the PHY layer, the fading parameters are $m_{P}=m_{S}=571$ $m_{P S}=2$, and all the associated thresholds are $8.5 \mathrm{~dB}$. The 572 normalized transmit power of the SU varies from 16 to $40 \mathrm{~dB}$ in 573 the three different cases associated with the normalized transmit 574 power of the PU, which are $P_{P}=22,20,18 \mathrm{~dB}$. The other 575 parameters are the same as in Table I. At the MAC layer, the 576 average packet arrival rate is $\lambda_{P}=0.5$ packet/TS, whereas the 577 delay tolerance is $T_{P}=5 \mathrm{TS}$.

It may be observed in Fig. 6 that the MASR of the SU 579 first increases upon increasing the normalized transmit power 580 $P_{S}$, since increasing $P_{S}$ improves the SINR performance of 581 the SU link, which results in an improved MASR. However, 582 beyond a specific point, the MASR is gradually reduced upon 583 further increasing $P_{S}$. That is because a higher $P_{S}$ imposes 584 an increased interference upon the PU link, which reduces the 585 PU's chance of emptying its own queues. As long as the PU 586 occupies the resources, this reduces the chances of the SU to 587 
588 access the system, and as a result, the MASR of the SU is 589 reduced. Given a higher transmit power $P_{P}$ of the PU, the 590 MASR of the SU is also increased, and the optimal power $P_{S}$ 591 maximizing the MASR of the SU becomes explicit in Fig. 6.

\section{CONCLUSION}

593 Our novel hybrid CR system amalgamated the interweave 594 and underlay paradigms for the sake of enhancing the chances 595 of the SU to access the system. The hybrid parameter $\varepsilon$ was 596 introduced for optimally blending the interweave and under597 lay paradigms, which was defined as the SU's probability of 598 accessing the system when the PU is still transmitting. Sev599 eral problems have been solved without violating the delay 600 constraints of the PU: 1) The scenario relying on realistic 601 imperfect sensing was considered. Under the assumption of 602 Nakagami- $m$ fading, we have modeled the service links by a 603 Poisson service process. 2) The optimal hybrid parameter of $604 \varepsilon^{*}$ was found for the MASR of the SU. 3) The most suitable 605 queue scheduling scheme $\left\{p_{i}^{*}, i=1,2, \ldots, N\right\}$ was found for 606 the sake of minimizing the OAD of the SU's multiple queues. 607 Our numerical results characterized the influence of the PHY608 and MAC-layer parameters on both the delay imposed and the 609 achievable average service rate of the SU. Several interesting 610 observations have been made: 1) Given different traffic loads 611 of the PU, different design strategies should be adopted for 612 the sake of enhancing the MASR of the SU. 2) If the traffic 613 load is high, our best strategy is to focus on improving the PU 614 link, which is capable of attaining a more substantial overall 615 performance gain, rather than improving the channel conditions 616 of the SU link. If the traffic load is light, improving the SU link 617 may achieve the best performance. Furthermore, improving the 618 reliability of the SU's sensing scheme is capable of achieving 619 the same overall performance gain as improving the PU link. 6203 ) When the delay tolerance tends to infinity, the performance 621 of the system becomes limited by the queue stability. 4) The 622 SU's OAD relying on our optimal scheme gets up to $27 \%$ 623 and $20 \%$ lower than that of the proportional and round-robin 624 schemes. 5) The optimal transmit power of the SU may be 625 found for the sake of maximizing the average service rate of 626 the SU.

627 In our future work, the shadowing effect imposed by the 628 wireless channel will also be taken into account. Furthermore, 629 the SU's performance will be studied to select the most suitable 630 transmit power.

\section{REFERENCES}

1] A. Goldsmith, S. A. Jafar, I. Maric, and S. Srinivasa, "Breaking spectrum gridlock with cognitive radios: An information theoretic perspective," Proc. IEEE, vol. 97, no. 5, pp. 894-914, May 2009.

[2] M. Nakagami, "The $m$-distribution-A general formula of intensity distribution of rapid fading," in Statistical Methods in Radio Wave Propagation. Oxford, U.K.: Pergamon, 1960, pp. 3-36.

[3] L. Hanzo, P. J. Cherriman, and J. Streit, Video Compression and Communications: From Basics to H.261, H.263, H.264, MPEG4 for DVB and HSDPA-Style Adaptive Turbo-Transceivers. Hoboken, NJ: Wiley, 2007.

[4] Q. Du and X. Zhang, "Statistical QoS provisionings for wireless unicast/ multicast of multi-layer video streams," IEEE J. Sel. Areas Commun., vol. 28, no. 3, pp. 420-433, Apr. 2010.
[5] O. Simeone, Y. Bar-Ness, and U. Spagnolini, "Stable throughput of cogni- 644 tive radios with and without relaying capability," IEEE Trans. Commun., 645 vol. 55, no. 12, pp. 2351-2360, Dec. 2007.

[6] X. Bao, P. Martins, T. Song, and L. Shen, "Stable throughput analysis of 647 multi-user cognitive cooperative systems," in Proc. IEEE GLOBECOM, 648 2010, pp. 1-5.

[7] A. A. El-Sherif, A. K. Sadek, and K. J. R. Liu, "Opportunistic multiple ac- 650 cess for cognitive radio networks," IEEE J. Sel. Areas Commun., vol. 29, 651 no. 4, pp. 704-714, Apr. 2011.

[8] J. Hu, L.-L. Yang, and L. Hanzo, "Appendix of the Submitted Paper "Max- 653 imum Average Service Rate and Optimal Queue Scheduling of Delay- 654 Constrained Hybrid Cognitive Radio in Nakagami Fading Channels," 655 Tech. Rep. [Online]. Available: http://eprints soton.ac.uk/340414/ 656

[9] A. Goldsmith, Wireless Communications. Cambridge, U.K.: Cambridge 657 Univ. Press, 2005.

[10] P. V. Mieghem, Performance Analysis of Communications Networks and 659 Systems. Cambridge, U.K.: Cambridge Univ. Press, 2006.

[11] V. Naware, G. Mergen, and L. Tong, "Stability and delay of finite-user 661 slotted ALOHA with multipacket reception," IEEE Trans. Inf. Theory, 662 vol. 51, no. 7, pp. 2636-2656, Jul. 2005.

663

[12] R. Rao and A. Ephremides, "On the stability of interacting queues in a 664 multiple-access system," IEEE Trans. Inf. Theory, vol. 34, no. 5, pp. 918- 665 930, Sep. 1988.

[13] W. Luo and A. Ephremides, "Stability of $N$ interacting queues in random 667 access systems," IEEE Trans. Inf. Theory, vol. 45, no. 5, pp. 1579-1587, 668 Jul. 1999.

[14] S. Boyd and L. Vandenberghe, Convex Optimization. Cambridge, U.K.: 670 Cambridge Univ. Press, 2004.

[15] I. S. Gradshteyn and I. M. Ryzhik, Table of Integrals, Series, and 672 Products, 7th ed. New York: Academic, 2007.

[16] L.-L. Yang Multicarrier Communications. Hoboken, NJ: Wiley, 674 Jan. 2009.

675

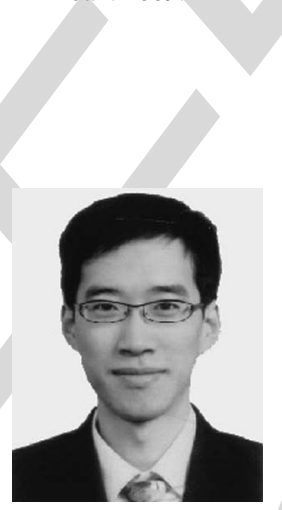

Jie Hu (S'11) received the B.Eng. degree in com- 676 munication engineering and the M.Eng. degree 677 in communication and information system from 678 Beijing University of Posts and Telecommunica- 679 tions, Beijing, China, in 2008 and 2011, respectively. 680 $\mathrm{He}$ is currently working toward the Ph.D. degree with 681 the Communication, Signal Processing and Control 682 Group, University of Southampton, Southampton, 683 U.K.

His research interests in wireless communica- 685 tions include cognitive radio and cognitive networks, 686 queuing analysis, resource allocation and scheduling, ad hoc wireless networks, 687 and mobile social networks.

688

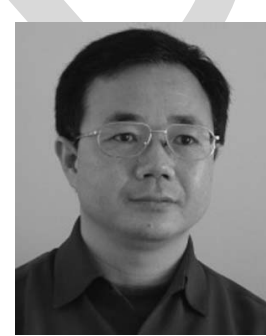

Lie-Liang Yang (M'98-SM'02) received the 689 B.Eng. degree in communications engineering from 690 Shanghai Tiedao University, Shanghai, China, 691 in 1988 and the M.Eng. and Ph.D. degrees in 692 communications and electronics from Northern 693 (Beijing) Jiaotong University, Beijing, China, in 694 1991 and 1997, respectively.

695

From June 1997 to December 1997, he was a Vis- 696 iting Scientist with the Institute of Radio Engineering 697 and Electronics, Academy of Sciences of the Czech 698 Republic, Prague, Czech Republic. Since December 699 1997, he has been with the University of Southampton, Southampton, U.K., 700 where he is currently a Professor with the School of Electronics and Computer 701 Science. He has published more than 240 research papers in journals and 702 conference proceedings, authored or coauthored three books, and published 703 several book chapters. Details about his publications can be found at http:// 704 www-mobile.ecs.soton.ac.uk/lly/. His research has covered a wide range of 705 topics in wireless communications, networking, and signal processing. $\quad 706$

Dr. Yang is currently an Associate Editor of the IEEE TRANSACTIONS ON 707 Vehicular TEChNOLOGY, the Journal of Communications and Networks, 708 and the Security and Communication Networks journal. 


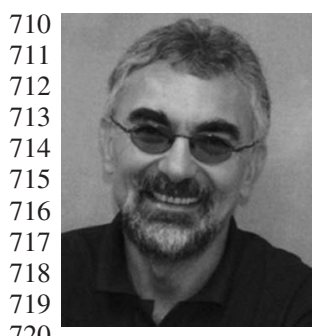

Lajos Hanzo (M'91-SM'92-F'04) received the M.S. degree (with first-class honors) in electronics and the Ph.D. degree from the Technical University of Budapest, Budapest, Hungary, in 1976 and 1983, respectively, the D.Sc. degree from the University of Southampton, Southampton, U.K., in 2004, and the "Doctor Honoris Causa" degree from the Technical University of Budapest in 2009.

During his 35-year career in telecommunications, he has held various research and academic posts in Hungary, Germany, and the U.K. Since 1986, he has 721 been with the School of Electronics and Computer Science, University of 722 Southampton, where he holds the Chair in Telecommunications. Since 2009, 723 he has been a Chaired Professor with Tsinghua University, Beijing, China. $724 \mathrm{He}$ is currently directing a 100-strong academic research team, working on a 725 range of research projects in the field of wireless multimedia communications 726 sponsored by industry; the Engineering and Physical Sciences Research Coun727 cil, U.K.; the European Information Society Technologies Program; and the 728 Mobile Virtual Centre of Excellence, U.K. He is an enthusiastic supporter of 729 industrial and academic liaison and offers a range of industrial courses. $\mathrm{He}$ 730 has successfully supervised $80 \mathrm{Ph}$.D. students, coauthored 20 John Wiley/IEEE 731 Press books on mobile radio communications totaling in excess of 10000 pages, 732 published more than 1250 research entries on IEEE Xplore, and presented 733 keynote lectures. For further information on research in progress and associated 734 publications, see http://www-mobile.ecs.soton.ac.uk/.

735 Dr. Hanzo is a Fellow of the Royal Academy of Engineering, U.K., a Fellow 736 of the Institution of Electrical Engineers, and a Governor of the IEEE Vehicular 737 Technology Society. He has been a Technical Program Committee Chair and a 738 General Chair for IEEE conferences. During 2008-2012, he was the Editor-in739 Chief of the IEEE Press. He has received a number of distinctions. 
AUTHOR QUERY

NO QUERY. 


\title{
, Maximum Average Service Rate and Optimal Queue 2 Scheduling of Delay-Constrained Hybrid Cognitive Radio in Nakagami Fading Channels
}

\author{
Jie Hu, Student Member, IEEE, Lie-Liang Yang, Senior Member, IEEE, and Lajos Hanzo, Fellow, IEEE
}

\begin{abstract}
5 Abstract-As a promising technique to improve achievable 6 bandwidth efficiency, cognitive radio (CR) has attracted substan7 tial research attention from both the academic and industrial com8 munities. To improve the performance attained by the secondary 9 user (SU), a novel hybrid CR system is proposed, which combines 10 the conventional interweave and underlay paradigms to enhance 11 the chance of the SU to access the spectrum. Queuing theory 12 is invoked in this paper to analyze the impact of the primary 13 user's maximum tolerable delay on the performance of the SU. 14 Multiple queues are assumed for the SU, which is engaged in 15 video communication. Apart from the Poisson traffic generation, 16 we also model the classic Nakagami- $\boldsymbol{m}$ fading channel as a Poisson 17 service process by utilizing the outage probability in the presence 18 of cochannel interference. We optimize both the hybrid inter19 weave/underlay procedure to maximize the average service rate $20 \mu_{S, \max }$ of the SU, as well as the queue's scheduling scheme, for the 21 sake of minimizing the overall average delay (OAD). As a result, 22 the OAD of the SU is reduced by up to $27 \%$ and $20 \%$, compared 23 with the proportion and round-robin schemes, respectively.
\end{abstract}

24 Index Terms-Hybrid cognitive radio (CR) system, maximum 25 average service rate (MASR) of secondary user (SU), multilayer 26 video streams (VSs), Nakagami- $m$ fading channel, optimal queue 27 scheduling scheme.

\section{INTRODUCTION}

29 WO kinds of customers are supported in cognitive radio 30 (CR), namely, the primary user (PU) and the secondary 31 user (SU). Furthermore, three main paradigms are consid32 ered for CR systems, namely, overlay, underlay, and inter33 weave paradigms [1]. Here, we focus our attention on the 34 interweave and underlay paradigms. According to the inter35 weave paradigm, the PUs are authorized to access the channel, 36 whereas the SUs are only able to access it when the PUs release 37 it. In the scenario that the PUs request resources, an SU's 38 session must be temporarily paused until the PUs complete 39 their transmission. In the interweave paradigm, SUs sense the 40 activities of PUs and clinch every possible opportunity to carry

Manuscript received June 21, 2012; revised November 15, 2012 and December 20, 2012; accepted December 27, 2012. This work was supported in part by the RC-UK's India-UK Advanced Technology Centre, by the European Union's Concerto Project, by the China-UK Science Bridge, and by the China Scholarship Council. The review of this paper was coordinated by Prof. C. Lin.

The authors are with the School of Electronics and Computer Science, University of Southampton, Southampton SO17 1BJ, U.K. (e-mail: jh10g11@ecs. soton.ac.uk; 1ly@ecs.soton.ac.uk; 1h@ecs.soton.ac.uk).

Color versions of one or more of the figures in this paper are available online at http://ieeexplore.ieee.org.

Digital Object Identifier 10.1109/TVT.2012.2237422

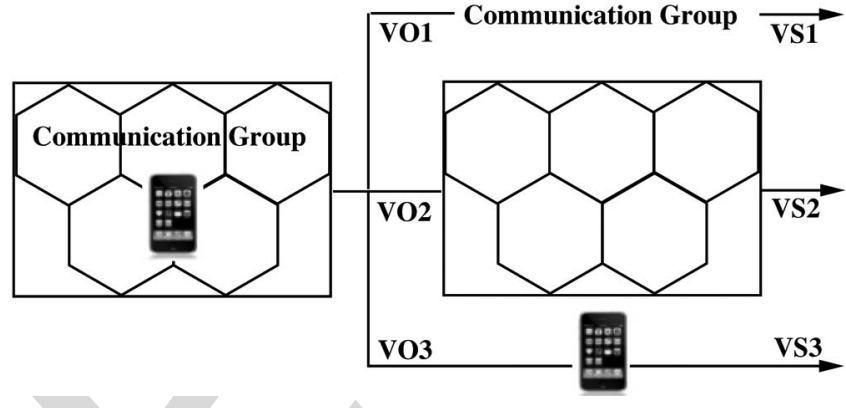

Fig. 1. Example of object-based MPEG-4 video transmission.

on with their own transmissions. By contrast, according to the 41 underlay paradigm, PUs and SUs are allowed to transmit their 42 data at the same time. An interference threshold is set up at 43 the PUs to guarantee that the PU's link is not unduly interfered 44 by SUs. Their transmission would only be terminated if the 45 interference imposed by SUs exceeds the threshold.

46

Although the transmission of PUs may be fully guaranteed 47 with the aid of the interweave paradigm, only limited op- 48 portunities may be offered to SUs to convey their own data. 49 Albeit SUs have improved chances of transmitting their own 50 information to the destination using the underlay paradigm, 51 the quality of service (QoS) of the PUs might not be as high 52 as for the interweave paradigm. Can we strike an attractive 53 compromise between these two paradigms so that the SUs have 54 more opportunities to access the channels while the PUs may 55 benefit from improved QoS? In this paper, a hybrid CR system 56 is invoked to achieve this goal. In our novel system, SUs first 57 detect the activity of PUs. If the channels are idle, the SUs 58 may access them to transmit their own data. By contrast, if 59 the channels are occupied by PUs, the SUs have a certain 60 probability of accessing the channels while ensuring that the 61 interference thresholds are not violated.

The Nakagami- $m$ channel model has the advantage of char- 63 acterizing a diverse range of multipath channels by a single 64 fading parameter $m$ [2]. Recently, supporting real-time video 65 services subject to specific QoS constraints has become one 66 of the essential requirements in wireless communications net- 67 works, where the video source is usually encoded into a number 68 of streams in the application layer, representing the base and 69 enhancement layers. An example of object-based MPEG-4 70 video transmission [3] is shown in Fig. 1, where different video 71 objects are encoded into different video streams (VSs). The 72 scheduling of the multilayer video queues at the SU's end 73 
74 deserves further attention for the sake of achieving the best 75 possible performance.

76 In [4], an adaptive resource-allocation scheme for multi77 layer VSs transmitted in wireless unicast/multicast scenarios is 78 proposed, which inspires our work. The maximum throughput 79 of an SU has been investigated in [5] under the interweave 80 paradigm by finding the optimal transmit power of the SU 81 in the scenario of a single queue conceived for the SU. In 82 [6], this research is extended to the multiple PU and single 83 SU CR system to explore the optimal transmit power and the 84 relaying probability at the SU. The stability region has been 85 already analyzed in the CR network supporting multiple PUs, 86 multiple relays, and multiple SUs [7]. In [5]-[7], the interweave 87 paradigm is employed, where the SUs have fewer opportunities 88 to access the resources. All these studies assumed, however, that 89 a single queue was set up for each of the users, which cannot 90 faithfully represent the characteristics of multimedia communi91 cation. Furthermore, all results have been generated assuming 92 queuing stability, where the specific QoS constraints such as 93 the delay tolerance of lip-synchronized video communications 94 were completely ignored.

95 Against this background, our novel contributions are as 96 follows.

97 1) We amalgamate the interweave and underlay paradigms 98 into a novel hybrid CR scheme characterized by the 99 parameter $0 \leq \varepsilon \leq 1$, which is zero when the pure inter100 weave paradigm is used and one for the pure underlay 101 paradigm.

102 2) The Nakagami- $m$ fading channel is used while modeling 103 the service as a Poisson process by deriving the closed117 including both the medium access control (MAC) and phys118 ical (PHY) layers, are described in Section II, whereas in 119 Section III, the related queuing analysis is carried out for both 120 the PU and the SU. In Section IV, the problem of finding the 121 optimal solution is formulated and solved, followed by our 122 numerical results in Section V. Finally, our conclusions are 123 offered in Section VI.

\section{SYSTEM MODEL}

125 Fig. 2 shows a CR system having a pair of PU source and 126 PU destination (PD), as well as a pair of SU source and SU 127 destination (SD). A novel hybrid CR policy is employed. Given 128 a spectrum band, the SU first senses the activity of the PU. If 129 this sensing process is reliable, the SU may access the spectrum

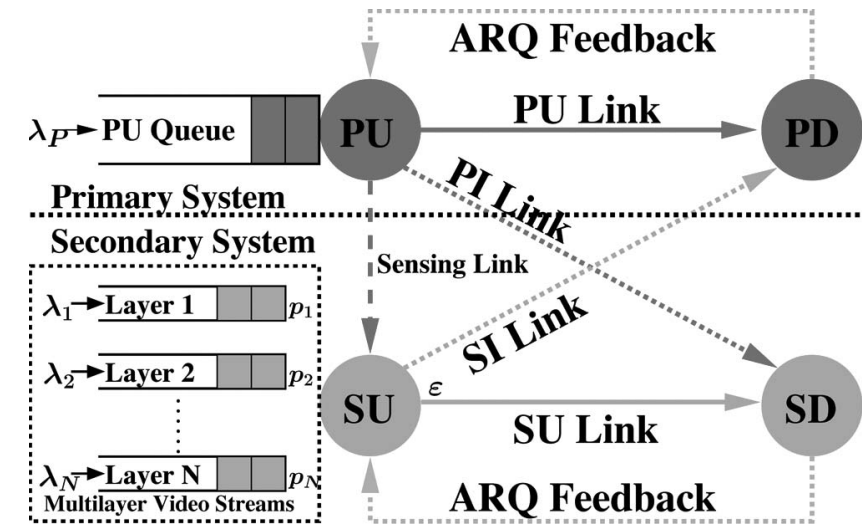

Fig. 2. System model with one PU and one SU sharing the same spectrum.

with a unity probability when the PU is idle. Furthermore, 130 the SU may access the spectrum with a probability of $0 \leq 131$ $\varepsilon \leq 1$, even if the PU is busy, provided that the PU's QoS is 132 still guaranteed. If, however, this sensing process failed, the 133 situation is reversed, and hence, the SU accesses the spectrum 134 with a probability of $\varepsilon$ when the PU is idle and with a unity 135 probability when the PU is busy. Hence, erroneous spectrum 136 sensing may lead to catastrophic performance degradation for 137 both the PU and the SU. We will also consider the practical 138 scenario of an erroneous spectrum-sensing decision and its 139 effect on the performance of the SU. Let us now consider the 140 details of the MAC and PHY layers.

\section{A. MAC-Layer Model}

Observe in Fig. 2 that a buffer is provided for the PU to store 143 the packets, which cannot be immediately transmitted. Again, 144 to support video communications for the SU, multiple buffers 145 are provided to accommodate the different-priority queues gen- 146 erated by multilayer video encoding.

The basic unit of time in our system is a time slot (TS). The 148 packets' arrival at the PU's buffer obeys the classic Poisson 149 process with a mean of $\lambda_{P}$ packets/TS. The QoS constraint 150 of the PU is the total delay imposed by both the transmission 151 and buffering delay, which should not exceed $T_{P}$, namely, the 152 maximum tolerable delay of the PU. The packets' arrival at 153 the buffer input of the SU also follows the Poisson process 154 with a mean of $\left\{\lambda_{n}, n=1,2, \ldots, N\right\}$ packets/TS. Regardless 155 of whether the PU is idle or busy, if the SU has already 156 successfully accessed the spectrum, we should determine the 157 probability of each queue at the SU completing its transmission 158 of the stored packets. These probabilities may be denoted 159 by $\left\{p_{n}, n=1,2, \ldots, N\right\}$. No specific QoS constraints are 160 imposed at the SU. However, our prime goal is to find the 161 optimal scheduling scheme and the associated hybrid parameter 162 $\varepsilon$, which minimizes the OAD of all the queues at the SU. 163

We assume that every packet transmitted by the PU or the 164 SU may be only successfully received by the corresponding 165 destination in a TS when the signal-to-interference-plus-noise 166 ratio (SINR) is above a specific threshold. In our system, 167 automatic repeat request (ARQ) with an unlimited number of 168 retransmissions is adopted to ensure that no packets are lost. 169 
170 Furthermore, we assume that the acknowledgements are always 171 successfully received by the transmitters.

172 Theorem 1: Given the successful reception probability $\mu$ of 173 a single packet during a TS in the ARQ-aided system, the 174 continuous transmit time obeys the exponential distribution 175 with the mean of $1 / \mu$.

176 Proof: The proof is provided in [8, App. A].

177 As a benefit of the ARQ mechanism, a packet's departure 178 from the buffer is synonymous to being served. The wireless 179 channel may be considered as a "server" in the queuing analy180 sis. As a result, the packets' departure process is also referred 181 to as the service process.

\section{B. PHY-Layer Model}

183 Observe from Fig. 2 that there are five links in this hybrid CR 184 system, namely, the PU link, the SU link, the sensing link, the 185 PI link imposing interference from the PU on the SD, and the 186 SI link inflicting interference from the SU upon the PD. In line 187 with [11], a multipacket reception (MPR) model is introduced 188 for the queuing analysis of the collision channel. We define the 189 corresponding conditional probabilities for the MPR model of 190 these five links.

$191 q_{\mathrm{PU} \mid\{\mathrm{PU}\}}^{(\mathrm{PD})}$ (or $q_{\mathrm{SU} \mid\{\mathrm{SU}\}}^{(\mathrm{SD})}$ ): Only the PU's (or SU's) packet is 192 successfully received at the PD (or SD) when only the PU 193 (or SU) transmits.

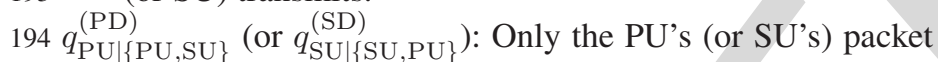
195 is successfully received at the PD (or SD) when both the $196 \quad$ PU and the SU transmit.

$197 q_{\mathrm{PU} \mid\{\mathrm{PU}\}}^{(\mathrm{SU})}$ : Only the PU's packet is successfully received at the 198 SU when the PU transmits.

199 Next, we will briefly highlight the derivation of these condi200 tional probabilities. The radio propagation between any pair of 201 nodes is assumed to be affected by the independent stationary 202 Nakagami- $m$ flat-fading channels $h_{i}(t)$ having $E\left[\left|h_{i}(t)\right|^{2}\right]=1$ 203 ( $t$ is the TS index). Hence, $Z=\left|h_{i}(t)\right|^{2}$ is a Gamma-distributed 204 random variable (r.v.) having a mean of 1, which may be 205 denoted by $Z \sim \operatorname{Gamma}(m, 1 / m)$ with the shape and scale 206 parameters of $m$ and $1 / m$, respectively. Given the probability 207 density function (pdf) $f_{Z}(z)$ of [9, eq. (3.39)], the tail dis208 tribution function (TDF) of $Z$ is formulated as $P(Z>z)=$ $209 \Gamma(m, m z) / \Gamma(m)$, where $\Gamma(\cdot, \cdot)$ represents the upper incomplete 210 Gamma function, and $m$ denotes the Nakagami- $m$ fading pa211 rameter [15].

212 After being divided by the noise power, the normalized trans213 mit power of the PU is defined as $P_{P}$, whereas the normalized 214 transmit power of the SU is defined as $P_{S}$. The propagation 215 path loss (PL) is denoted by $\Omega_{i}$, and the SINR threshold that 216 has to be exceeded for successful packet reception is denoted by $217 \beta_{i}$, while again, the Nakagami- $m$ fading parameter is denoted 218 by $m_{i}$, where $i$ represents "P" for the PU link, "S" for the SU 219 link, "PI" for the PI link, "SI" for the SI link, and "PS" for the 220 sensing link spanning from the PU to the SU.

221 According to the TDF of the r.v. $Z$, we express the con222 ditional probabilities of $q_{\mathrm{PU} \mid\{\mathrm{PU}\}}^{(\mathrm{PD})}, q_{\mathrm{SU} \mid\{\mathrm{SU}\}}^{(\mathrm{SD})}$, and $q_{\mathrm{PU} \mid\{\mathrm{PU}\}}^{(\mathrm{SU})}$ in the MPR model in the absence of interference thresholds as 223 follows:

$$
\begin{aligned}
q_{\mathrm{PU} \mid\{\mathrm{PU}\}}^{(\mathrm{PD})} & =P\left[\frac{\left|h_{P}(t)\right|^{2} P_{P}}{\Omega_{P}}>\beta_{P}\right] \\
& =\Gamma\left(m_{P}, \frac{m_{P} \beta_{P} \Omega_{P}}{P_{P}}\right) / \Gamma\left(m_{P}\right) \\
q_{\mathrm{SU} \mid\{\mathrm{SU}\}}^{(\mathrm{SD})} & =P\left[\frac{\left|h_{S}(t)\right|^{2} P_{S}}{\Omega_{S}}>\beta_{S}\right] \\
& =\Gamma\left(m_{S}, \frac{m_{S} \beta_{S} \Omega_{S}}{P_{S}}\right) / \Gamma\left(m_{S}\right) \\
q_{\mathrm{PU} \mid\{\mathrm{PU}\}}^{(\mathrm{SU})} & =P\left[\frac{\left|h_{\mathrm{PS}}(t)\right|^{2} P_{P}}{\Omega_{\mathrm{PS}}}>\beta_{\mathrm{PS}}\right] \\
& =\frac{\Gamma\left(m_{\mathrm{PS}}, m_{\mathrm{PS}} \beta_{\mathrm{PS}} \Omega_{\mathrm{PS}} / P_{P}\right)}{\Gamma\left(m_{\mathrm{PS}}\right)} .
\end{aligned}
$$

We introduce another two Gamma-distributed r.v.'s, namely, 225 $X \sim \operatorname{Gamma}\left(m_{X}, 1 / m_{X}\right)$ with a pdf of $f_{X}(x)$ and $Y \sim 226$ $\operatorname{Gamma}\left(m_{Y}, 1 / m_{Y}\right)$ with a pdf of $f_{Y}(y)$. Then, the problem 227 of solving $q_{\mathrm{PU} \mid\{\mathrm{PU}, \mathrm{SU}\}}^{(\mathrm{PD})}$ and $q_{\mathrm{SU} \mid\{\mathrm{SU}, \mathrm{PU}\}}^{(\mathrm{SD})}$ becomes equivalent to 228 solving the probability problem of $P[X>A+B Y]$. Unfortu- 229 nately, a closed-form solution cannot be derived for the general 230 situation. Nonetheless, we may obtain the following results for 231 two special cases:

$$
\begin{aligned}
& P[X>A+B Y] \\
& =\left\{\begin{array}{rr}
\Phi(A, B), & \text { if } m_{X} \text { is a positive integer } \\
& m_{Y} \text { can be any real number } \\
\Phi^{\prime}(A, B), & \text { if } m_{Y} \text { is a positive integer } \\
& m_{X} \text { can be any real number. }
\end{array}\right. \\
& \left\{\begin{aligned}
\Phi(A, B)= & \left(\frac{m_{Y}}{m_{X} B+m_{Y}}\right)^{m_{Y}} e^{-m_{X} A} \\
& \cdot \sum_{n=0}^{m_{X}-1} \sum_{k=0}^{n} \frac{m_{X}^{n} B^{k} A^{n-k}}{k !(n-k) !\left(m_{X} B+m_{Y}\right)^{k}} \frac{\Gamma\left(m_{Y}+k\right)}{\Gamma\left(m_{Y}\right)} \\
\Phi^{\prime}(A, B)= & \frac{\Gamma\left(m_{X}, m_{X} A\right)}{\Gamma\left(m_{X}\right)}-\sum_{n=0}^{m_{Y}-1} \sum_{k=0}^{n}\left(\begin{array}{l}
n \\
k
\end{array}\right)\left(\frac{m_{Y}}{B}\right)^{n} \\
& \cdot \frac{e^{m_{Y} \frac{A}{B}(-A)^{n-k} m_{X}^{m_{X}}}}{n ! \Gamma\left(m_{X}\right)} \cdot \frac{\Gamma\left[k+m_{X}, A\left(m_{X}+m_{Y} / B\right)\right]}{\left(m_{X}+m_{Y} / B\right)^{k+m_{X}}} .
\end{aligned}\right.
\end{aligned}
$$

For a detailed derivation of $\Phi(A, B)$ and $\Phi^{\prime}(A, B)$, see 233 [8, App. B]. Given this result, we may write down the other 234 two conditional probabilities when subjected to the cochannel 235 interference thresholds at the PD and the SD as follows:

236

$$
\begin{aligned}
& q_{\mathrm{PU} \mid\{\mathrm{PU}, \mathrm{SU}\}}^{(\mathrm{PD})} \\
& \quad=P\left[\frac{\left|h_{P}(t)\right|^{2} P_{P} / \Omega_{P}}{1+\left|h_{\mathrm{SI}}(t)\right|^{2} P_{S} / \Omega_{\mathrm{SI}}}>\beta_{P}\right] \\
& \quad=P\left[\left|h_{P}(t)\right|^{2}>\frac{\beta_{P} \Omega_{P}}{P_{P}}+\frac{\beta_{P} P_{S} \Omega_{P}}{P_{P} \Omega_{\mathrm{SI}}}\left|h_{\mathrm{SI}}(t)\right|^{2}\right] \\
& \quad= \begin{cases}\Phi\left(\frac{\beta_{P} \Omega_{P}}{P_{P}}, \frac{\beta_{P} P_{S} \Omega_{P}}{P_{P} \Omega_{\mathrm{SI}}}\right), & \text { if } m_{P} \text { is a positive integer } \\
\Phi^{\prime}\left(\frac{\beta_{P} \Omega_{P}}{P_{P}}, \frac{\beta_{P} P_{S} \Omega_{P}}{P_{P} \Omega_{S I}}\right), & \text { if } m_{S I} \text { is a positive integer }\end{cases}
\end{aligned}
$$




$$
\begin{aligned}
& q_{\mathrm{SU} \mid\{\mathrm{SU}, \mathrm{PU}\}}^{(\mathrm{SD})} \\
& \quad=P\left[\frac{\left|h_{S}(t)\right|^{2} P_{S} / \Omega_{S}}{1+\left|h_{\mathrm{PI}}(t)\right|^{2} P_{P} / \Omega_{\mathrm{PI}}}>\beta_{S}\right] \\
& =P\left[\left|h_{S}(t)\right|^{2}>\frac{\beta_{S} \Omega_{S}}{P_{S}}+\frac{\beta_{S} P_{P} \Omega_{S}}{P_{S} \Omega_{\mathrm{PI}}}\left|h_{\mathrm{PI}}(t)\right|^{2}\right] \\
& \quad= \begin{cases}\Phi\left(\frac{\beta_{S} \Omega_{S}}{P_{S}}, \frac{\beta_{S} P_{P} \Omega_{S}}{P_{S} \Omega_{\mathrm{PI}}}\right), & \text { if } m_{S} \text { is a positive integer } \\
\Phi^{\prime}\left(\frac{\beta_{S} \Omega_{S}}{P_{S}}, \frac{\beta_{S} P_{P} \Omega_{S}}{P_{S} \Omega_{\mathrm{PI}}}\right), & \text { if } m_{\mathrm{PI}} \text { is a positive integer. }\end{cases}
\end{aligned}
$$

\section{QUEUING ANALYSIS}

238 The following tasks will be accomplished here: 1) The classic $239 \mathrm{M} / \mathrm{M} / 1$ queuing is revisited. 2) The average service rate of the $240 \mathrm{PU}$ and the SU is attained for the original benchmark system. $2413)$ A new system is defined for characterizing the interdepen242 dence of the PU's and SU's queues, and the corresponding 243 average service rate of the PU and the SU is rederived.

\section{A. $M / M / 1$ Queuing System}

245 In the $M / M / 1$ queuing system, only a single server is in246 voked by the system. The packets' interarrival time follows 247 the exponential distribution having a parameter $\lambda$, whereas the 248 packets' interdeparture time obeys the same distribution having 249 a parameter $\mu$. An infinite buffer is assumed for guaranteeing 250 that every single packet may be successfully transmitted, i.e., 251 without any packet loss. According to [10], we can express 252 the probability of the queue being empty and the total average 253 delay, including the transmission and buffering time, as follows:

$$
P[Q=0]=1-\lambda / \mu, \quad T=1 /(\mu-\lambda), \quad \text { where } \quad \lambda<\mu
$$

254 and we denote the random queue length and the total average 255 delay by $Q$ and $T$, respectively.

\section{B. PU Link}

257 According to the MAC-layer protocol of our hybrid CR 258 system, the PU has the highest priority to access the channel. If 259 the PU's queue is not empty, the PU would occupy the channel 260 for its transmission, and the packet would be removed from the 261 head of the queue when it is successfully received by the PD. 262 However, the PU would suffer from the interference imposed 263 by the activity of the SU. The service process of a PU's single 264 packet follows the Bernoulli distribution. In analogy to the 265 successful packet departure probability in the Bernoulli trial, 266 the average service rate of the Poisson service process may be 267 formulated for the PU link as

$$
\begin{aligned}
\mu_{P}= & P\left\{Q_{S}=0\right\} \cdot q_{\mathrm{PU} \mid\{\mathrm{PU}\}}^{(\mathrm{PD})}+P\left\{Q_{S} \neq 0\right\} \\
& \cdot\left(q_{\mathrm{PU} \mid\{\mathrm{PU}\}}^{(\mathrm{SU})} \cdot\left(q_{\mathrm{PU} \mid\{\mathrm{PU}\}}^{(\mathrm{PD})} \cdot(1-\varepsilon)+q_{\mathrm{PU} \mid\{\mathrm{PU}, \mathrm{SU}\}}^{(\mathrm{PD})} \cdot \varepsilon\right)\right. \\
& +\left(1-q_{\mathrm{PU} \mid\{\mathrm{PU}\}}^{(\mathrm{SU})}\right) \cdot q_{\mathrm{PU} \mid\{\mathrm{PU}, \mathrm{SU}\}}^{(\mathrm{PD})}
\end{aligned}
$$

where $Q_{S}$ represents the random queue length of the SU. Given 268 the average service rate $\mu_{P}$, the average arrival rate $\lambda_{P}$, and the 269 QoS constraint stipulating that the total delay should not exceed 270 the maximum delay tolerance $T_{P}$, we infer from (4) that the 271 condition $0 \leq \lambda_{P} \leq \mu_{P}-1 / T_{P}$ must be obeyed.

\section{SU Link}

According to the MAC-layer protocol of the SU, before 274 accessing the channel, the SU carries out spectrum sensing first. 275 When the channel is released by the PU, after reliable sensing, 276 the SU would transmit its own packets with a probability 277 of unity (otherwise, with a probability of $\varepsilon$ ), which implies 278 inefficient resource usage. When the channel is still occupied 279 by the PU, following reliable sensing, the SU would transmit 280 its own packets with a probability of $\varepsilon$ (otherwise, with a 281 probability of unity), which imposes strong interference on the 282 PD. It is vital to guarantee that the transmission of the SU does 283 not force the PU to violate its delay tolerance. We derive the 284 probability of a packet being served, which is also identical to 285 the average service rate $\mu_{S}$ of the SU's Poisson service process 286 expressed as

$$
\begin{aligned}
\mu_{S}= & q_{\mathrm{SU} \mid\{\mathrm{SU}\}}^{(\mathrm{SD})} \cdot P\left[Q_{P}=0\right] \\
& \cdot\left[q_{\mathrm{PU} \mid\{\mathrm{PU}\}}^{(\mathrm{SU})}+\left(1-q_{\mathrm{PU} \mid\{\mathrm{PU}\}}^{(\mathrm{SU})}\right) \cdot \varepsilon\right] \\
& +q_{\mathrm{SU} \mid\{\mathrm{SU}, \mathrm{PU}\}}^{(\mathrm{SD})} \cdot P\left[Q_{P} \neq 0\right] \\
& \cdot\left[q_{\mathrm{PU} \mid\{\mathrm{PU}\}}^{(\mathrm{SU})} \cdot \varepsilon+\left(1-q_{\mathrm{PU} \mid\{\mathrm{PU}\}}^{(\mathrm{SU})}\right)\right]
\end{aligned}
$$

where $Q_{P}$ represents the random queue length of the PU.

\section{Stochastic Dominance Principle}

It may be shown from (5) and (6) that the average service 290 rates of the SU and the PU depend on each other's queue sizes. 291 Since these queues interact with each other, the average rate of 292 the individual service processes cannot be directly determined. 293 For the sake of circumventing this problem, the stochastic 294 dominance principle of [12] is invoked to assist our analysis. 295

The concept of a so-called "dominant system" was defined 296 in [12] by allowing a set of terminals having no packets in their 297 transmit-buffer to continue transmitting hypothetical dummy 298 packets. In this manner, the queues in the dominant system 299 stochastically dominate the queues in the original system. This 300 dominant system is defined here.

301

1) If we have $Q_{S}=0$ and $Q_{P}=0$, the SU transmits dummy 302 packets with a unity probability.

303

2) If $Q_{S}=0$ and $Q_{P} \neq 0$, the SU transmits dummy packets 304 with a probability $\varepsilon$.

305

Note that the hypothetical dummy packets are introduced 306 only for the sake of facilitating our performance analysis and 307 for finding closed-form solutions to the optimum scheduling 308 factor $\varepsilon$, but in reality, no dummy packets are assumed to 309 be transmitted in the practical systems considered. As shown 310 in [13], given the same initial conditions, the queuing per- 311 formance of the dominant system transmitting hypothetical 312 
313 dummy packets is capable of providing a tight approximation 314 of the queuing performance of the original system. Since in 315 practical systems no dummy packets are actually transmitted, 316 no extra interference will be imposed on the system, and no 317 extra power is required for their transmissions.

318 In the context of the extended dominant system, $\left\{Q_{S} \neq 0\right\}$ 319 has a unity probability. Hence, (5) can be rewritten as

$$
\begin{array}{r}
\mu_{P}=q_{\mathrm{PU} \mid\{\mathrm{PU}\}}^{(\mathrm{SU})} \cdot\left(q_{\mathrm{PU} \mid\{\mathrm{PU}\}}^{(\mathrm{PD})} \cdot(1-\varepsilon)+q_{\mathrm{PU} \mid\{\mathrm{PU}, \mathrm{SU}\}}^{(\mathrm{PD})} \cdot \varepsilon\right) \\
+\left(1-q_{\mathrm{PU} \mid\{\mathrm{PU}\}}^{(\mathrm{SU})}\right) \cdot q_{\mathrm{PU} \mid\{\mathrm{PU}, \mathrm{SU}\}}^{(\mathrm{PD})}
\end{array}
$$

320 and the condition $0 \leq \lambda_{P} \leq \mu_{P}-1 / T_{P}$ is also rewritten as

$$
\begin{aligned}
& 0 \leq \lambda_{P} \leq q_{\mathrm{PU} \mid\{\mathrm{PU}\}}^{(\mathrm{SU})} q_{\mathrm{PU} \mid\{\mathrm{PU}\}}^{(\mathrm{PD})} \\
&+\left(1-q_{\mathrm{PU} \mid\{\mathrm{PU}\}}^{(\mathrm{SU})}\right) q_{\mathrm{PU} \mid\{\mathrm{PU}, \mathrm{SU}\}}^{(\mathrm{PD})}-1 / T_{P} \\
& \quad-q_{\mathrm{PU} \mid\{\mathrm{PU}\}}^{(\mathrm{SU})}\left(q_{\mathrm{PU} \mid\{\mathrm{PU}\}}^{(\mathrm{PD})}-q_{\mathrm{PU} \mid\{\mathrm{PU}, \mathrm{SUh}\}}^{(\mathrm{PD})}\right) \varepsilon .
\end{aligned}
$$

321 According to (4), we may represent the probabilities of the PU's 322 queue being not empty and empty by $\lambda_{P}$ and $\mu_{P}$, respectively, 323 as shown in (7). Hence, we may rewrite (6) as

$$
\begin{aligned}
\mu_{S}=q_{\mathrm{SU} \mid\{\mathrm{SU}\}}^{\mathrm{SD}} & q_{\mathrm{PU} \mid\{\mathrm{PU}\}}^{(\mathrm{SU})}+q_{\mathrm{SU} \mid\{\mathrm{SU}\}}^{\mathrm{SD}}\left(1-q_{\mathrm{PU} \mid\{\mathrm{PU}\}}^{(\mathrm{SU})}\right) \varepsilon \\
-\frac{\lambda_{P}}{\mu_{P}} & {\left[q_{\mathrm{SU} \mid\{\mathrm{SU}\}}^{\mathrm{SD}} q_{\mathrm{PU} \mid\{\mathrm{PU}\}}^{(\mathrm{SU})}+q_{\mathrm{SU} \mid\{\mathrm{SU}\}}^{\mathrm{SD}}\right.} \\
& \times\left(1-q_{\mathrm{PU} \mid\{\mathrm{PU}\}}^{(\mathrm{SU})}\right) \varepsilon-q_{\mathrm{SU} \mid\{\mathrm{SU}, \mathrm{PU}\}}^{\mathrm{SD}} q_{\mathrm{PU} \mid\{\mathrm{PU}\}}^{(\mathrm{SU})} \varepsilon \\
& \left.-q_{\mathrm{SU} \mid\{\mathrm{SU}, \mathrm{PU}\}}^{\mathrm{SD}}\left(1-q_{\mathrm{PU} \mid\{\mathrm{PU}\}}^{(\mathrm{SU})}\right)\right]
\end{aligned}
$$

324 To simplify the derivations in the next section, some sim325 ple notations are introduced as follows: $C=q_{\mathrm{PU} \mid\{\mathrm{PU}\}}^{(\mathrm{PD})}, D=$ $326 q_{\mathrm{PU} \mid\{\mathrm{PU}, \mathrm{SU}\}}^{(\mathrm{PD})}, E=q_{\mathrm{SU} \mid\{\mathrm{SU}\}}^{(\mathrm{SD})}, F=q_{\mathrm{SU} \mid\{\mathrm{SU}, \mathrm{PU}\}}^{(\mathrm{SD})}$, and $S=q_{\mathrm{PU} \mid\{\mathrm{PU}\}}^{(\mathrm{SU})}$

\section{7}

\section{Problem Formulation and Solutions}

328 Here, the following two problems are solved: 1) finding the 329 optimal $\varepsilon^{*}$ for maximizing the average service rate $\mu_{S, \max }$ of 330 the SU and 2) finding the optimal queue scheduling scheme $331\left\{p_{n}^{*}, n=1,2, \ldots, N\right\}$ to minimize the OAD associated with $332 \mu_{S, \max }$.

\section{A. MASR of the $S U$}

334 The optimal parameter $\varepsilon^{*}$ will be found for the sake of 335 maximizing the average service rate of the SU without violating 336 the delay tolerance of the PU. The solution can be found by 337 constructing the problem $\mathbf{P 1}$ as

$$
\begin{gathered}
\arg \max _{\varepsilon} \mu_{S}(\varepsilon) \\
0 \leq \varepsilon \leq \xi=\frac{S C+(1-S) D-\lambda_{P}-1 / T_{P}}{S(C-D)} \leq 1
\end{gathered}
$$

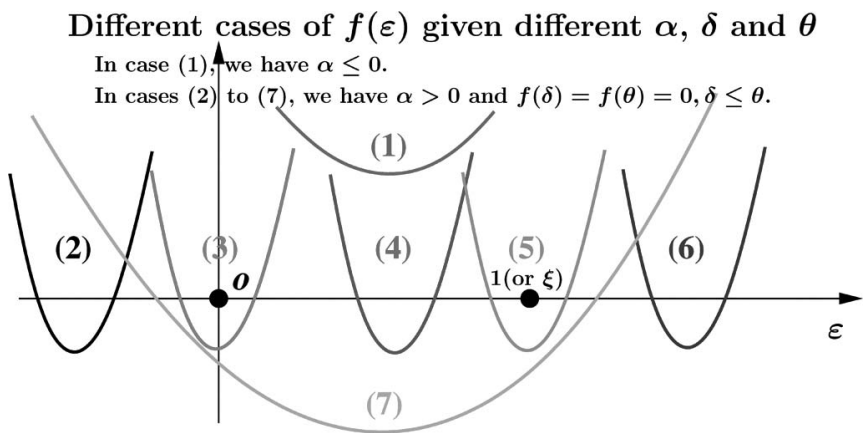

Fig. 3. Different cases of $f(\varepsilon)$ given different $\alpha, \delta$, and $\theta$.

where the function $\mu_{S}(\varepsilon)$ is defined by (9), whereas the inequal- 338 ity condition in (10) is imposed for the sake of satisfying the 339 specific delay tolerance of the PU, which is derived from (8). 340

To solve P1, we differentiate $\mu_{S}(\varepsilon)$ with respect to $\varepsilon, 341$ yielding

$$
\frac{d \mu_{S}}{d \varepsilon}=\frac{f(\varepsilon)}{[S C+(1-S) D-S(C-D) \varepsilon]^{2}}
$$

where we define $f(\varepsilon)=E(1-S)[S C+(1-S) D-S(C-343$ $D) \varepsilon]^{2}-\lambda_{P} \alpha$ and $\alpha=S(C-D)(E-F)+D E(1-S)-344$ $F S D$. Since the denominator of (11) is always positive, we 345 can simply consider $f(\varepsilon)$ instead, which is a quadratic function 346 having the shape shown in Fig. 3.

347

Let us consider the constraint shown in (8) more closely. 348 Given $0 \leq \varepsilon \leq 1$, the right side of (8) reaches its minimum 349 value of $\left(D-1 / T_{P}\right)$ when we have $\varepsilon=1$. Therefore, if we 350 have $\lambda_{P} \leq D-1 / T_{P}, \varepsilon$ may assume any arbitrary value rang- 351 ing from 0 to 1 . By contrast, if $\lambda_{P}>D-1 / T_{P}$, then $\varepsilon$ must 352 be selected from the range determined by (10) in the context of 353 the specific $\lambda_{P}$ value.

Different scenarios have to be considered for finding the 355 optimal values of $\varepsilon^{*}$, which lead to the maximum average 356 service rate (MASR) $\mu_{S}(\varepsilon)$ of the SU formulated in (10). $\quad 357$

Case $1\left(\lambda_{P} \leq D-1 / T_{P}\right)$ : In this case, as aforementioned, 358 $\varepsilon$ might assume any arbitrary value ranging from 0 to 1 . Under 359 this assumption, the problem may be further divided into the 360 following two subcases, which will assist us in finding $\mu_{S, \max } .361$

Subcase 1-1 $(\alpha \leq 0)$ : When $\alpha \leq 0$, the $f(\varepsilon)$ curve obeys the 362 relative position shown by case (1) at the top of Fig. 3, in which 363 case there are no real-valued solutions to $f(\varepsilon)=0$. Hence, 364 provided that $E(1-S)>0$ holds, $f(\varepsilon)$ is always higher than 365 0 , implying that $\mu_{S}(\varepsilon)$ is a monotonically increasing function. 366 Therefore, we have $\varepsilon^{*}=1$ and $\mu_{S, \max }=\mu_{S}(1)$.

Subcase 1-2 $(\alpha>0)$ : Second, when $\alpha>0$, there are two 368 real-valued solutions to $f(\varepsilon)=0$, which are denoted by $\delta$ and 369 $\theta$, respectively, i.e.,

$$
\begin{aligned}
\delta= & \frac{1}{S(C-D)} \\
& {[S C+(1-D)} \\
& -\sqrt{\left.\frac{\lambda_{P}[S(C-D)(E-F)+D E(1-S)-F S D]}{E(1-S)}\right]}
\end{aligned}
$$




$$
\begin{aligned}
\theta= & \frac{1}{S(C-D)} \\
& {[S C+(1-D)} \\
& +\sqrt{\left.\frac{\lambda_{P}[S(C-D)(E-F)+D E(1-S)-F S D]}{E(1-S)}\right]}
\end{aligned}
$$

371 where we have $\delta \leq \theta$. In this case, depending on the values of $\delta$ 372 and $\theta$, the relative position of the $f(\varepsilon)$ curve corresponds to one 373 of the scenarios (2)-(7) shown in Fig. 3, which are specifically 374 considered in the following.

a) If $\delta \leq \theta<0$, the $f(\varepsilon)$ curve obeys the positioning of case (2) in Fig. 3, where $f(\varepsilon)>0$ holds within the range of $0 \leq \varepsilon \leq 1$, implying that $\mu_{S}(\varepsilon)$ is a monotonically increasing function of $\varepsilon$. Hence, $\varepsilon^{*}=1$ maximizes the average service rate $\mu_{S}(\varepsilon)$, which is $\mu_{S, \max }=\mu_{S}(1)$.

b) If $\delta<0$ and $0 \leq \theta<1$, the $f(\varepsilon)$ curve obeys the positioning of case (3) in Fig. 3. Hence, $f(\varepsilon) \leq 0$ holds within the range of $0 \leq \varepsilon \leq \theta$, which implies that $\mu_{S}(\varepsilon)$ is a monotonically decreasing function of $\varepsilon$. However, within the range of $\theta<\varepsilon \leq 1, f(\varepsilon)>0$ holds, and hence, $\mu_{S}(\varepsilon)$ is a monotonically increasing function of $\varepsilon$. In this situation, the optimal value of $\varepsilon^{*}$ is either 0 or 1 . Hence, we have $\varepsilon^{*}=\arg \max _{\varepsilon \in\{0,1\}}\left\{\mu_{S}(\varepsilon)\right\}$, and the MASR becomes $\mu_{S, \max }=\max \left\{\mu_{S}(0), \mu_{S}(1)\right\}$.

c) If $0 \leq \delta<\theta<1$, the $f(\varepsilon)$ curve corresponds to case (4) in Fig. 3. Therefore, $f(\varepsilon) \geq 0$ holds within the range of $0 \leq \varepsilon \leq \delta$, implying that $\mu_{S}(\varepsilon)$ is a monotonically increasing function of $\varepsilon$. Within the range of $\delta<\varepsilon \leq$ $\theta$, however, $f(\varepsilon) \leq 0$ holds, which suggests that $\mu_{S}(\varepsilon)$ is a monotonically decreasing function of $\varepsilon$. Finally, in the range of $\theta<\varepsilon \leq 1, f(\varepsilon)>0$ is satisfied, which indicates that $\mu_{S}(\varepsilon)$ becomes a monotonically increasing function of $\varepsilon$ again. According to the preceding analysis, the pair of potential values that may maximize $\mu_{S}(\varepsilon)$ are $\varepsilon=\delta$ and $\varepsilon=1$. Hence, the optimal value of $\varepsilon^{*}$ is $\varepsilon^{*}=\arg \max _{\varepsilon \in\{\delta, 1\}}\left\{\mu_{S}(\varepsilon)\right\}$, and the MASR is, hence, $\mu_{S, \max }=\max \left\{\mu_{S}(\delta), \mu_{S}(1)\right\}$.

d) If $0 \leq \delta<1$ and $\theta \geq 1$, which results in an $f(\varepsilon)$ curve corresponding to case (5) in Fig. 3, $f(\varepsilon) \geq 0$ holds within the range of $0 \leq \varepsilon \leq \delta$, which implies that $\mu_{S}(\varepsilon)$ is a monotonically increasing function of $\varepsilon$. Within the range of $\delta<\varepsilon \leq 1$, however, $f(\varepsilon)<0$ holds, which implies that $\mu_{S}(\varepsilon)$ is a monotonically decreasing function of $\varepsilon$. Jointly considering these two situations, we conclude that $\varepsilon^{*}=\delta$ maximizes the average service rate $\mu(\varepsilon)$, which is $\mu_{S, \max }=\mu_{S}(\delta)$.

e) If $\delta \geq 1$, we have encountered case (6) of Fig. 3. Correspondingly, $f(\varepsilon) \geq 0$ holds within the range of $0 \leq \varepsilon \leq$ 1 , which implies that $\mu_{S}(\varepsilon)$ is a monotonically increasing function of $\varepsilon$. Hence, $\varepsilon^{*}=1$ maximizes the average service rate of $\mu_{S}(\varepsilon)$, which is $\mu_{S, \max }=\mu_{S}(1)$.

f) Finally, if $\delta<0<1 \leq \theta, f(\varepsilon)$ is reminiscent of case (7) of Fig. 3. Consequently, $f(\varepsilon) \leq 0$ holds within the range of $0 \leq \varepsilon \leq 1$, which implies that $\mu_{S}(\varepsilon)$ is a monotoni- cally decreasing function of $\varepsilon$. Hence, $\varepsilon^{*}=0$ maximizes 419 the average service rate $\mu_{S}(\varepsilon)$, which is $\mu_{S, \max }=\mu_{S}(0) .420$

Case $2\left(\lambda_{P}>D-1 / T_{P}\right)$ : If $\lambda_{P}>D-1 / T_{P}$, then $\varepsilon$ may 421 assume any arbitrary value ranging from 0 to $\xi$, where the value 422 of $\xi$ is restricted by the value of $\lambda_{P}$ according to (10). Following 423 a similar train of thought, by simply substituting "1" in Case 1424 by " $\xi$," we have the following conclusions, which correspond 425 to the cases (1)-(7) in Fig. 3, as we discussed earlier. 426

1) If $\alpha \leq 0$ (see case (1) in Fig. 3), or if $\alpha>0$ and $\theta<0427$ (see case (2) in Fig. 3), or if $\alpha>0$ and $\xi \leq \delta$ (see case (6) 428 in Fig. 3), then we have $\varepsilon^{*}=\xi$, and the MASR becomes 429 $\mu_{S, \max }=\mu_{S}(\xi)$.

2) If $\alpha>0, \delta<0$, and $0 \leq \theta<\xi$ (see case (3) in Fig. 3), 431 then we have $\varepsilon^{*}=\arg \max _{\varepsilon \in\{0, \xi\}}\left\{\mu_{S}(\varepsilon)\right\}$, and the 432 MASR becomes $\mu_{S, \max }=\max \left\{\mu_{S}(0), \mu_{S}(\xi)\right\}$.

433

3) If $\alpha>0$ and $0 \leq \delta<\theta<\xi$ (see case (4) in Fig. 3), then 434 we have $\varepsilon^{*}=\arg \max _{\varepsilon \in\{\delta, \xi\}}\left\{\mu_{S}(\varepsilon)\right\}$, and the MASR is 435 $\mu_{S, \max }=\max \left\{\mu_{S}(\delta), \mu_{S}(\xi)\right\}$.

436

4) If $\alpha>0$ and $0 \leq \delta<\xi \leq \theta$ (see case (5) in Fig. 3), then 437 $\varepsilon^{*}=\delta$, and the MASR is $\mu_{S, \max }=\mu_{S}(\delta)$.

438

5) If $\alpha>0, \delta<0$, and $0<\xi \leq \theta$ (see case (7) in Fig. 3), then 439 $\varepsilon^{*}=0$, and the MASR is formulated as $\mu_{S, \max }=\mu_{S}(0) .440$

\section{B. Optimal Queue Scheduling of the $S U$}

441

Let us now further detail the derivation of the optimal queue 442 scheduling scheme $\left\{p_{n}^{*}, n=1,2, \ldots, N\right\}^{1}$ conceived for min- 443 imizing the OAD of the SU's queues. Given (4), the average 444 delay of each queue may be formulated as

445

$$
\operatorname{Delay}_{n}=1 /\left(p_{n} \cdot \mu_{S}(\varepsilon)-\lambda_{n}\right), \quad n=1,2, \ldots, N
$$

where $p_{n}$ is the probability of the SU's $n$th queue transmitting 446 its stored packets, and $\lambda_{n}$ is the packets' average arrival rate. 447

Given the associated MASR $\mu_{S, \text { max }}$ of the SU, we construct 448 the second problem $\mathbf{P 2}$, i.e.

449

$$
\begin{aligned}
& \arg \min _{\left\{p_{1}, p_{2}, \ldots, p_{N}\right\}}\left\{\frac{1}{N} \sum_{n=1}^{N} \frac{1}{p_{n} \cdot \mu_{S, \max }-\lambda_{n}}\right\} \\
& \text { subject to } \sum_{n=1}^{N} p_{n}=1, \quad \lambda_{n}-p_{n} \cdot \mu_{S, \max }<0 .
\end{aligned}
$$

Then, we may readily show the following.

1) The objective function (15) is convex over $\left\{p_{n}, n=451\right.$ $1,2, \ldots, N\}$.

2) The first equality constraint in (16) is affine over 453 $\left\{p_{n}, n=1,2, \ldots, N\right\}$, whereas the second inequality 454 constraint in (16) is convex over $\left\{p_{n}, n=1,2, \ldots, N\right\} .455$

Hence, $\mathbf{P} \mathbf{2}$ is a convex problem [14], and the optimal solution 456 may be obtained with the aid of the Lagrangian optimization 457 method while additionally exploiting the Karush-Kuhn-Tucker 458 (KKT) conditions [14], which were proposed for solving 459 convex optimization problems similar to ours under an inequal- 460 ity constraint.

461

\footnotetext{
${ }^{1}$ The optimal shceduling scheme is fully specified when the probability $p_{n}$ of transmitting the stream $n$ is determined.
} 
TABLE I

PHY-LAYER PARAMETER SETTING

\begin{tabular}{|l|r|r|r|r|}
\hline & $\mathrm{PL}$ & Threshold & Fading & Norm Power \\
\hline PU Link & $\Omega_{P}=5 \mathrm{~dB}$ & $\beta_{P}=13.9$ or $8.5 \mathrm{~dB}$ & $m_{P}=1$ or 2 & $P_{P}=26 \mathrm{~dB}$ \\
\hline SU Link & $\Omega_{S}=5 \mathrm{~dB}$ & $\beta_{S}=13.9$ or $8.5 \mathrm{~dB}$ & $m_{S}=1$ or 2 & $P_{S}=26 \mathrm{~dB}$ \\
\hline PI/SI Link & $\Omega_{P I / S I}=15 \mathrm{~dB}$ & No threshold & $m_{S I / P I}=1$ & $P_{P / S}=26 \mathrm{~dB}$ \\
\hline Sensing Link & $\Omega_{P S}=2 \mathrm{~dB}$ & $\beta_{P S}=13.9$ or $8.5 \mathrm{~dB}$ & $m_{P S}=1$ or 2 & $P_{P}=26 \mathrm{~dB}$ \\
\hline
\end{tabular}

462

$$
\begin{aligned}
J=\frac{1}{N} \sum_{n=1}^{N} \frac{1}{p_{n} \mu_{S, \max }-\lambda_{n}} \\
\quad+\sum_{n=1}^{N} \varphi_{n}\left(\lambda_{n}-p_{n} \mu_{S, \max }\right)+\eta\left(\sum_{n=1}^{N} p_{n}-1\right)
\end{aligned}
$$

463 where $\eta \geq 0$ and $\varphi_{n} \geq 0, n=1,2, \ldots, N$ are the Lagrangian 464 multipliers associated with the two conditions of (16), respec465 tively. Then, the optimal queue scheduling scheme $\left\{p_{n}^{*}, n=\right.$ $4661,2, \ldots, N\}$ and the Lagrangian multipliers of the optimization 467 problem P2 satisfy the following KKT conditions [14]:

$$
\left\{\begin{array}{l}
\left.\frac{\partial J}{\partial p_{n}}\right|_{p_{n}=p_{n}^{*}}=\frac{-\mu_{S, \max }}{N\left(p_{n}^{*} \mu_{S, \max }-\lambda_{n}\right)^{2}}-\varphi_{n}^{*} \mu_{S, \max }+\eta^{*}=0 \\
\left.\frac{d J}{d \eta}\right|_{p_{n}=p_{n}^{*}}=\sum_{n=1}^{N} p_{n}^{*}-1=0 \\
\varphi_{n}^{*}\left(\lambda_{n}-p_{n}^{*} \mu_{S, \max }\right)=0, \quad \varphi_{n}^{*} \geq 0 \text { and } \eta^{*} \geq 0
\end{array}\right.
$$

468 where $n=1,2, \ldots, N$. Naturally, according to the second con469 straint of (16), the only solution satisfying the third line of (18) 470 is $\varphi_{n}^{*}=0$. Substituting $\varphi_{n}^{*}=0$ into the first line of (18), we 471 derive $p_{n}^{*}$ as

$$
p_{n}^{*}=\frac{1}{\sqrt{N \eta^{*} \mu_{S, \max }}}+\frac{\lambda_{n}}{\mu_{S, \max }} .
$$

472 Then, substituting (19) into the second line of (18), we arrive at

$$
\eta^{*}=N \cdot \mu_{S, \max } /\left(\mu_{S, \max }-\sum_{n=1}^{N} \lambda_{n}\right)^{2}
$$

473 Finally, substituting $\eta^{*}$ of (20) into (19), we arrive at the optimal 474 queue scheduling scheme characterized by

$$
p_{n}^{*}=\frac{\mu_{S, \max }-\sum_{n=1}^{N} \lambda_{n}+N \lambda_{n}}{N \cdot \mu_{S, \max }} .
$$

\section{5 \\ V. PERFormance AnAlysis}

476 In our numerical results, we link the PHY-layer parameters to 477 a realistic orthogonal frequency-division multiplexing scheme 478 communicating over Nakagami- $m$ fading channels without 479 channel coding. We define the SINR threshold of the receiver 480 for the sake of guaranteeing that the bit error ratio of the system 481 is not higher than $10^{-2}$. Hence, the threshold is determined 482 by the channel model having different fading parameters. For 483 example, according to [16, Fig. 5-5], if $m=1$, the threshold 484 at the receiver is $13.86 \mathrm{~dB}$, whereas the threshold is $8.50 \mathrm{~dB}$ 485 for $m=2$.

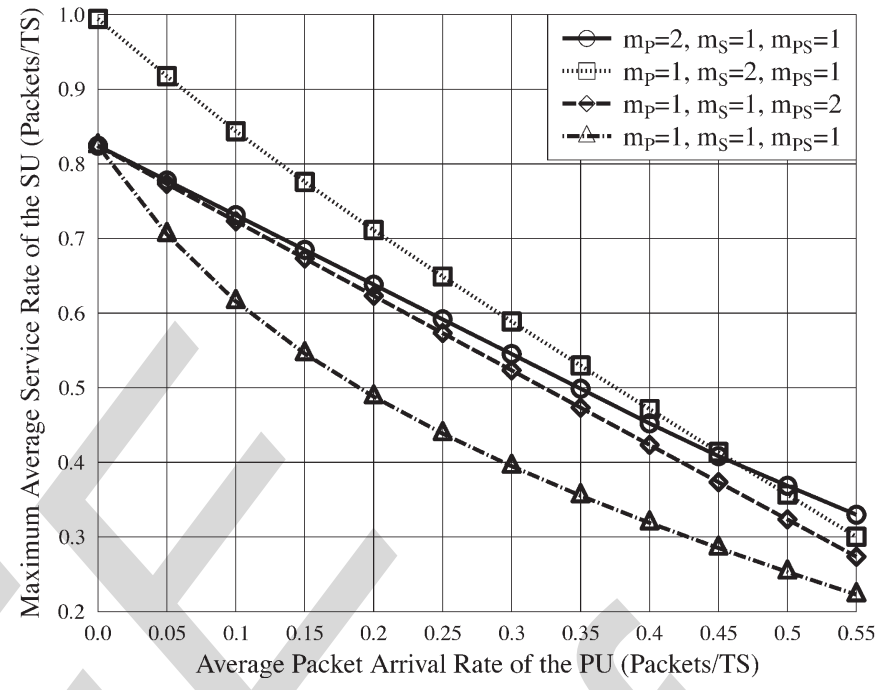

Fig. 4. Service rate of the SU versus the average packets arrival rate $\lambda_{P}$ of the PU parameterized by the fading parameters.

We assume that the SU has five different queuing buffers 486 in the MAC layer, where we set the average packet arrival 487 rate of each queue, for example, to $0.01,0.02,0.03,0.04$, and 488 0.05 packet/TS, respectively.

Two other queue scheduling schemes are used as benchmark- 490 ers, namely, the round-robin scheme, where every queue of the 491 SU has the same probability of transmitting packets stored in 492 the buffer, and the proportional scheme, where the transmission 493 probabilities are arranged to be proportional to the average 494 arrival rates at the buffers.

A. Impact of the PU'S Average Packet Arrival Rate

496

In the PHY layer, the PL, the SINR threshold of the receiver 497 associated with the Nakagami- $m$ fading parameters, and the 498 transmit power are set according to Table I. More particu- 499 larly, there are four cases for the Nakagami- $m$ parameters of 500 the PU, the SU, and the sensing links, namely, 1) $m_{P}=1,501$ $m_{S}=1$, and $m_{P S}=1$; 2) $m_{P}=2, m_{S}=1$, and $m_{P S}=1 ; 502$ 3) $m_{P}=1, m_{S}=2$, and $m_{P S}=1$; and 4) $m_{P}=1, m_{S}=1,503$ and $m_{P S}=2$.

In the MAC layer, the delay tolerance of the PU is $T_{P}=505$ $5 \mathrm{TS}$. We vary the average packet arrival rate $\lambda_{P}$ from 0.1 to 506 0.5 packet/TS for characterizing its impacts on the MASR and 507 the OAD in conjunction with three different queue scheduling 508 schemes for the SU.

It may be readily shown from Fig. 4 that $\mu_{S, \max }$ lin- 510 early decays upon increasing $\lambda_{P}$. This is plausible since a 511 higher $\lambda_{P}$ results in an increased traffic load for the PU link, 512 which, in turn, results in the PU's prolonged occupation of the 513 
514 spectral band. This reduces the SU's chance of transmitting its 515 own packets, which is reflected by the reduction of $\mu_{S, \max }$. 516 Upon increasing the Nakagami- $m$ parameters of the PU, SU, 517 and sensing links, respectively, the MASR is improved. If 518 we consider the higher $m_{P}$ of the PU link, it is shown in 519 Fig. 4 that the data stream of the PU may be transmitted more 520 promptly and the duration of the PU's spectrum occupation 521 would be shortened. Consequently, the SU's increased chances 522 of transmitting its own data results in an increased MASR. 523 Hence, the MASR is substantially improved. If we consider the 524 higher $m_{S}$ of the SU link characterized in Fig. 4, the MASR is 525 also directly improved. For higher $m_{P S}$ values, the MASR of 526 Fig. 4 would be also improved due to the fact that the spectrum527 sensing decisions become more reliable.

528 We may infer the following observations from Fig. 4 depend529 ing on the traffic load of the PU. We have the best performance 530 gain upon improving the SU link if the PU's traffic load is 531 lower than 0.45 packet/TS. By contrast, if the traffic load is 532 higher than 0.45 packet/TS, improving the PU link may provide 533 the best performance. Upon comparing the improvements of 534 the sensing and PU links shown in Fig. 4, when the PU's 535 traffic load is lower than 0.1 packet/TS, we infer that improving 536 the sensing link may provide the same performance gain as 537 improving the PU link. However, when the traffic load is higher 538 than 0.1 packet/TS, improving the PU link is more influential. 539 These observations may motivate us to design a more effective 540 strategy for the sake of improving the performance of the SU, 541 given different traffic loads of the PU. For example, given a 542 light PU traffic load, improving the SU link is our best option 543 to enhance overall performance. Furthermore, a reliable sensing 544 strategy conceived for the SU may achieve the same overall 545 performance as an improved PU link. By contrast, for a high 546 traffic load, researchers should focus on directly improving the 547 PU link.

\section{B. Impact of the PU'S Delay Tolerance on the $S U$}

549 Here, we consider the Nakagami- $m$ parameters of $m_{P}=$ $550 m_{S}=m_{P S}=2$, and all the associated SINR thresholds are $5518.5 \mathrm{~dB}$, where the transmit power of the PU and the SU are $552 P_{P}=P_{S}=18 \mathrm{~dB}$, whereas the others are the same as in 553 Table I. We vary the delay tolerance $T_{P}$ from 3 to 8 TS for 554 the sake of investigating its impacts on the OAD in conjunction 555 with three different queue scheduling schemes. Two scenarios, 556 namely, $\lambda_{P}=0.4$ and 0.5 packet/TS, are considered.

557 Observe furthermore in Fig. 5 that the SU benefits from 558 increasing $T_{P}$ and that a reduced $\lambda_{P}$ decreases the OAD. The 559 asymptotic values of the OAD for these three schemes may 560 be determined when $T_{P}$ tends to infinity. In Fig. 5, we focus 561 our attention on the comparison of three different scheduling 562 schemes. It can be seen that our proposed optimal scheme 563 (OPT) performs better than the conventional schemes. The 564 higher the traffic load of the PU, i.e., the higher $\lambda_{P}$ and 565 lower $T_{P}$, the more substantial the advantage of our optimal 566 scheme becomes. For example, for $\lambda_{P}=0.5$ packet/slot and $567 T_{P}=3 \mathrm{TS}$, our scheme has a $27 \%$ or $20 \%$ lower OAD than 568 the proportional (PRO) or the round-robin (R-R) schemes, 569 respectively, when considering the SU's queues.

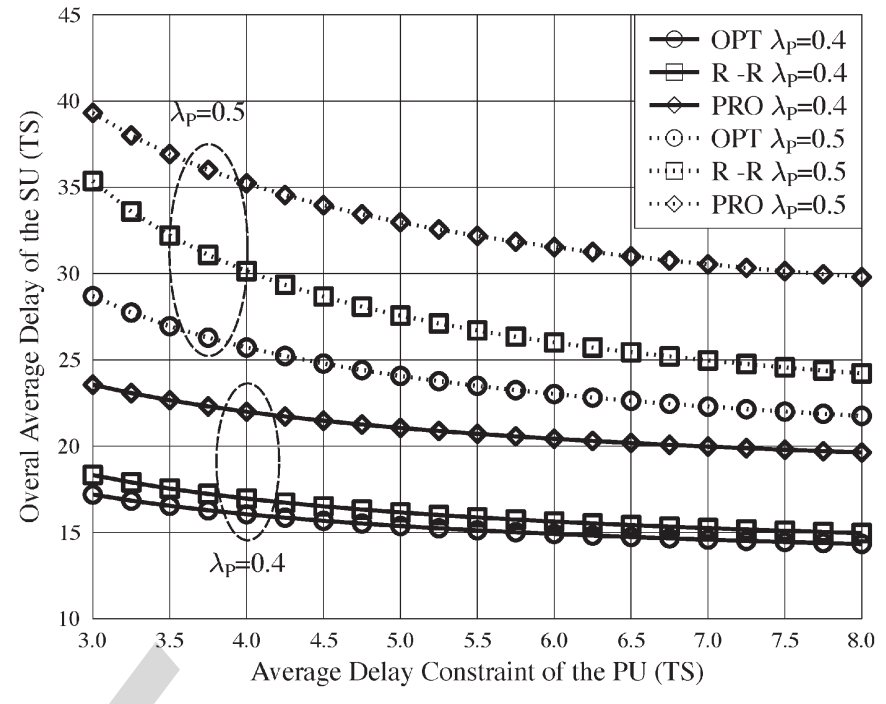

Fig. 5. Delay of the SU versus the delay tolerant $T_{P}$ of the PU parameterized by the average packets arrival rate $\lambda_{P}$.

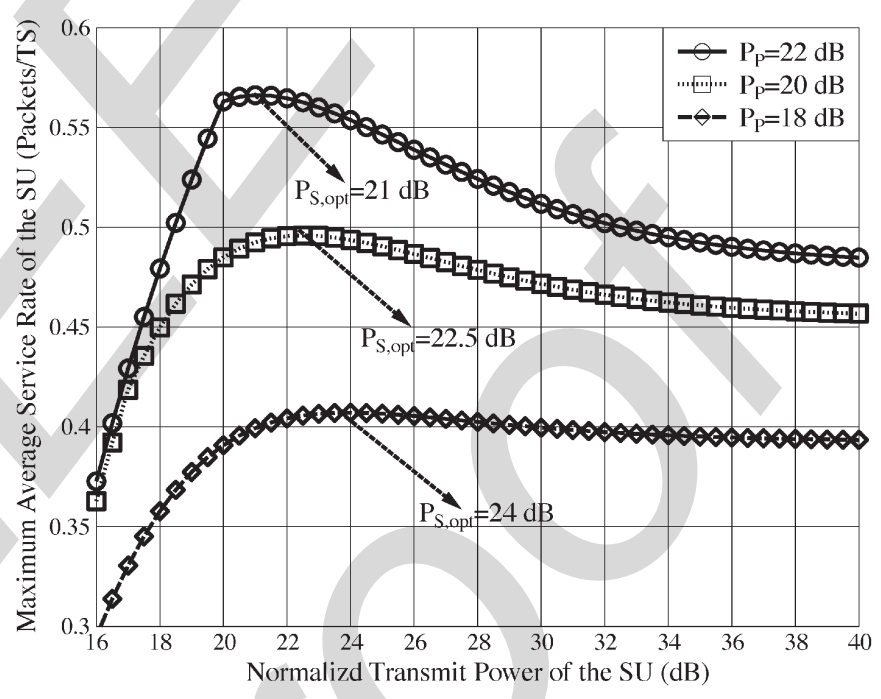

Fig. 6. Service rate versus the normalized transmit power of the SU parameterized by the normalized transmit power of the PU.

C. Impact of the SU's Normalized Transmit Power

In the PHY layer, the fading parameters are $m_{P}=m_{S}=571$ $m_{P S}=2$, and all the associated thresholds are $8.5 \mathrm{~dB}$. The 572 normalized transmit power of the SU varies from 16 to $40 \mathrm{~dB}$ in 573 the three different cases associated with the normalized transmit 574 power of the PU, which are $P_{P}=22,20,18 \mathrm{~dB}$. The other 575 parameters are the same as in Table I. At the MAC layer, the 576 average packet arrival rate is $\lambda_{P}=0.5$ packet/TS, whereas the 577 delay tolerance is $T_{P}=5 \mathrm{TS}$.

It may be observed in Fig. 6 that the MASR of the SU 579 first increases upon increasing the normalized transmit power 580 $P_{S}$, since increasing $P_{S}$ improves the SINR performance of 581 the SU link, which results in an improved MASR. However, 582 beyond a specific point, the MASR is gradually reduced upon 583 further increasing $P_{S}$. That is because a higher $P_{S}$ imposes 584 an increased interference upon the PU link, which reduces the 585 PU's chance of emptying its own queues. As long as the PU 586 occupies the resources, this reduces the chances of the SU to 587 
588 access the system, and as a result, the MASR of the SU is 589 reduced. Given a higher transmit power $P_{P}$ of the PU, the 590 MASR of the SU is also increased, and the optimal power $P_{S}$ 591 maximizing the MASR of the SU becomes explicit in Fig. 6.

\section{CONCLUSION}

593 Our novel hybrid CR system amalgamated the interweave 594 and underlay paradigms for the sake of enhancing the chances 595 of the SU to access the system. The hybrid parameter $\varepsilon$ was 596 introduced for optimally blending the interweave and under597 lay paradigms, which was defined as the SU's probability of 598 accessing the system when the PU is still transmitting. Sev599 eral problems have been solved without violating the delay 600 constraints of the PU: 1) The scenario relying on realistic 601 imperfect sensing was considered. Under the assumption of 602 Nakagami- $m$ fading, we have modeled the service links by a 603 Poisson service process. 2) The optimal hybrid parameter of $604 \varepsilon^{*}$ was found for the MASR of the SU. 3) The most suitable 605 queue scheduling scheme $\left\{p_{i}^{*}, i=1,2, \ldots, N\right\}$ was found for 606 the sake of minimizing the OAD of the SU's multiple queues. 607 Our numerical results characterized the influence of the PHY608 and MAC-layer parameters on both the delay imposed and the 609 achievable average service rate of the SU. Several interesting 610 observations have been made: 1) Given different traffic loads 611 of the PU, different design strategies should be adopted for 612 the sake of enhancing the MASR of the SU. 2) If the traffic 613 load is high, our best strategy is to focus on improving the PU 614 link, which is capable of attaining a more substantial overall 615 performance gain, rather than improving the channel conditions 616 of the SU link. If the traffic load is light, improving the SU link 617 may achieve the best performance. Furthermore, improving the 618 reliability of the SU's sensing scheme is capable of achieving 619 the same overall performance gain as improving the PU link. 6203 ) When the delay tolerance tends to infinity, the performance 621 of the system becomes limited by the queue stability. 4) The 622 SU's OAD relying on our optimal scheme gets up to $27 \%$ 623 and $20 \%$ lower than that of the proportional and round-robin 624 schemes. 5) The optimal transmit power of the SU may be 625 found for the sake of maximizing the average service rate of 626 the SU.

627 In our future work, the shadowing effect imposed by the 628 wireless channel will also be taken into account. Furthermore, 629 the SU's performance will be studied to select the most suitable 630 transmit power.

\section{REFERENCES}

1] A. Goldsmith, S. A. Jafar, I. Maric, and S. Srinivasa, "Breaking spectrum gridlock with cognitive radios: An information theoretic perspective," Proc. IEEE, vol. 97, no. 5, pp. 894-914, May 2009.

[2] M. Nakagami, "The $m$-distribution-A general formula of intensity distribution of rapid fading," in Statistical Methods in Radio Wave Propagation. Oxford, U.K.: Pergamon, 1960, pp. 3-36.

[3] L. Hanzo, P. J. Cherriman, and J. Streit, Video Compression and Communications: From Basics to H.261, H.263, H.264, MPEG4 for DVB and HSDPA-Style Adaptive Turbo-Transceivers. Hoboken, NJ: Wiley, 2007.

[4] Q. Du and X. Zhang, "Statistical QoS provisionings for wireless unicast/ multicast of multi-layer video streams," IEEE J. Sel. Areas Commun., vol. 28, no. 3, pp. 420-433, Apr. 2010.
[5] O. Simeone, Y. Bar-Ness, and U. Spagnolini, "Stable throughput of cogni- 644 tive radios with and without relaying capability," IEEE Trans. Commun., 645 vol. 55, no. 12, pp. 2351-2360, Dec. 2007.

[6] X. Bao, P. Martins, T. Song, and L. Shen, "Stable throughput analysis of 647 multi-user cognitive cooperative systems," in Proc. IEEE GLOBECOM, 648 2010, pp. 1-5.

[7] A. A. El-Sherif, A. K. Sadek, and K. J. R. Liu, "Opportunistic multiple ac- 650 cess for cognitive radio networks," IEEE J. Sel. Areas Commun., vol. 29, 651 no. 4, pp. 704-714, Apr. 2011.

[8] J. Hu, L.-L. Yang, and L. Hanzo, "Appendix of the Submitted Paper "Max- 653 imum Average Service Rate and Optimal Queue Scheduling of Delay- 654 Constrained Hybrid Cognitive Radio in Nakagami Fading Channels," 655 Tech. Rep. [Online]. Available: http://eprints soton.ac.uk/340414/ 656

[9] A. Goldsmith, Wireless Communications. Cambridge, U.K.: Cambridge 657 Univ. Press, 2005.

[10] P. V. Mieghem, Performance Analysis of Communications Networks and 659 Systems. Cambridge, U.K.: Cambridge Univ. Press, 2006.

[11] V. Naware, G. Mergen, and L. Tong, "Stability and delay of finite-user 661 slotted ALOHA with multipacket reception," IEEE Trans. Inf. Theory, 662 vol. 51, no. 7, pp. 2636-2656, Jul. 2005.

663

[12] R. Rao and A. Ephremides, "On the stability of interacting queues in a 664 multiple-access system," IEEE Trans. Inf. Theory, vol. 34, no. 5, pp. 918- 665 930, Sep. 1988.

[13] W. Luo and A. Ephremides, "Stability of $N$ interacting queues in random 667 access systems," IEEE Trans. Inf. Theory, vol. 45, no. 5, pp. 1579-1587, 668 Jul. 1999.

[14] S. Boyd and L. Vandenberghe, Convex Optimization. Cambridge, U.K.: 670 Cambridge Univ. Press, 2004.

[15] I. S. Gradshteyn and I. M. Ryzhik, Table of Integrals, Series, and 672 Products, 7th ed. New York: Academic, 2007.

[16] L.-L. Yang Multicarrier Communications. Hoboken, NJ: Wiley, 674 Jan. 2009.

675

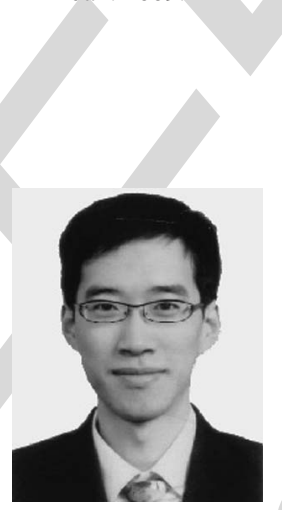

Jie Hu (S'11) received the B.Eng. degree in com- 676 munication engineering and the M.Eng. degree 677 in communication and information system from 678 Beijing University of Posts and Telecommunica- 679 tions, Beijing, China, in 2008 and 2011, respectively. 680 $\mathrm{He}$ is currently working toward the Ph.D. degree with 681 the Communication, Signal Processing and Control 682 Group, University of Southampton, Southampton, 683 U.K.

His research interests in wireless communica- 685 tions include cognitive radio and cognitive networks, 686 queuing analysis, resource allocation and scheduling, ad hoc wireless networks, 687 and mobile social networks.

688

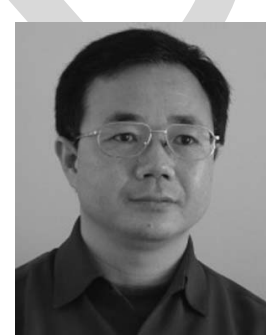

Lie-Liang Yang (M'98-SM'02) received the 689 B.Eng. degree in communications engineering from 690 Shanghai Tiedao University, Shanghai, China, 691 in 1988 and the M.Eng. and Ph.D. degrees in 692 communications and electronics from Northern 693 (Beijing) Jiaotong University, Beijing, China, in 694 1991 and 1997, respectively.

695

From June 1997 to December 1997, he was a Vis- 696 iting Scientist with the Institute of Radio Engineering 697 and Electronics, Academy of Sciences of the Czech 698 Republic, Prague, Czech Republic. Since December 699 1997, he has been with the University of Southampton, Southampton, U.K., 700 where he is currently a Professor with the School of Electronics and Computer 701 Science. He has published more than 240 research papers in journals and 702 conference proceedings, authored or coauthored three books, and published 703 several book chapters. Details about his publications can be found at http:// 704 www-mobile.ecs.soton.ac.uk/lly/. His research has covered a wide range of 705 topics in wireless communications, networking, and signal processing. $\quad 706$

Dr. Yang is currently an Associate Editor of the IEEE TRANSACTIONS ON 707 Vehicular TEChNOLOGY, the Journal of Communications and Networks, 708 and the Security and Communication Networks journal. 


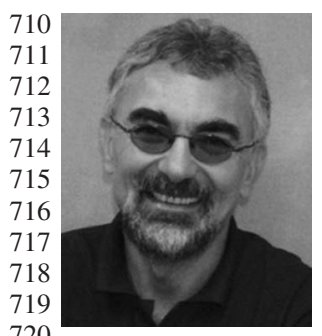

Lajos Hanzo (M'91-SM'92-F'04) received the M.S. degree (with first-class honors) in electronics and the Ph.D. degree from the Technical University of Budapest, Budapest, Hungary, in 1976 and 1983, respectively, the D.Sc. degree from the University of Southampton, Southampton, U.K., in 2004, and the "Doctor Honoris Causa" degree from the Technical University of Budapest in 2009.

During his 35-year career in telecommunications, he has held various research and academic posts in Hungary, Germany, and the U.K. Since 1986, he has 721 been with the School of Electronics and Computer Science, University of 722 Southampton, where he holds the Chair in Telecommunications. Since 2009, 723 he has been a Chaired Professor with Tsinghua University, Beijing, China. $724 \mathrm{He}$ is currently directing a 100-strong academic research team, working on a 725 range of research projects in the field of wireless multimedia communications 726 sponsored by industry; the Engineering and Physical Sciences Research Coun727 cil, U.K.; the European Information Society Technologies Program; and the 728 Mobile Virtual Centre of Excellence, U.K. He is an enthusiastic supporter of 729 industrial and academic liaison and offers a range of industrial courses. $\mathrm{He}$ 730 has successfully supervised $80 \mathrm{Ph}$.D. students, coauthored 20 John Wiley/IEEE 731 Press books on mobile radio communications totaling in excess of 10000 pages, 732 published more than 1250 research entries on IEEE Xplore, and presented 733 keynote lectures. For further information on research in progress and associated 734 publications, see http://www-mobile.ecs.soton.ac.uk/.

735 Dr. Hanzo is a Fellow of the Royal Academy of Engineering, U.K., a Fellow 736 of the Institution of Electrical Engineers, and a Governor of the IEEE Vehicular 737 Technology Society. He has been a Technical Program Committee Chair and a 738 General Chair for IEEE conferences. During 2008-2012, he was the Editor-in739 Chief of the IEEE Press. He has received a number of distinctions. 
AUTHOR QUERY

NO QUERY. 